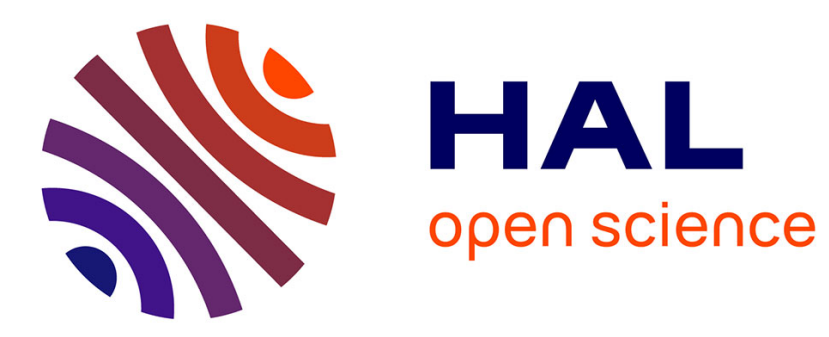

\title{
Identification of nonlinear dynamic models of electrostatically actuated MEMS
}

Céline Casenave, Emmanuel Montseny, Henri Camon

\section{To cite this version:}

Céline Casenave, Emmanuel Montseny, Henri Camon. Identification of nonlinear dynamic models of electrostatically actuated MEMS. Control Engineering Practice, 2010, 18 (8), pp.954-969. 10.1016/j.conengprac.2010.04.002 . hal-00833046

\section{HAL Id: hal-00833046 \\ https://hal.science/hal-00833046}

Submitted on 11 Jun 2013

HAL is a multi-disciplinary open access archive for the deposit and dissemination of scientific research documents, whether they are published or not. The documents may come from teaching and research institutions in France or abroad, or from public or private research centers.
L'archive ouverte pluridisciplinaire HAL, est destinée au dépôt et à la diffusion de documents scientifiques de niveau recherche, publiés ou non, émanant des établissements d'enseignement et de recherche français ou étrangers, des laboratoires publics ou privés. 


\title{
Identification of Nonlinear Dynamic Models of Electrostatically Actuated MEMS
}

\author{
Céline Casenave $^{\mathrm{a}, \mathrm{b}}$, Emmanuel Montseny ${ }^{\mathrm{a}, \mathrm{b}}$, Henri Camon ${ }^{\mathrm{a}, \mathrm{b}}$ \\ ${ }^{a}$ CNRS; LAAS; 7 avenue du colonel Roche, F-31077 Toulouse, France. \\ ${ }^{b}$ Université de Toulouse; UPS, INSA, INP, ISAE; LAAS; F-31077 Toulouse, France.
}

\begin{abstract}
This paper focuses on the identification of nonlinear dynamic models for physical systems such as electrostatically actuated micro-electro-mechanical systems (MEMS). The proposed approach consists in transforming, by means of suitable global operations, the input-output differential model in such a way that the new equivalent formulation is well adapted to the identification problem, thanks to the following properties: first, the linearity with respect to the parameters to be identified is preserved, second, the continuous dependence on noise measurements is restored. Consequently, a simple least-square resolution can be used, in such a way that some of the difficulties classically encountered with identification methods are by-passed. The method is implemented on real measurement data from a physical system.
\end{abstract}

Key words: Dynamic Models; Parameters Identification; Least-squares Identification; Time-continuous Identification; Electrostatically Actuated Micro-Electro-Mechanical Systems

\section{Introduction}

This paper focuses on the identification of nonlinear dynamic models for physical systems such as MEMS from measurement data associated with known inputs. One of the main reasons which makes identification an important step when working with such micro-systems is that physical modelling in general does not permit to get very reliable models, useful for example for control (Zhu et al. (2007); Liao et al. (2004); Sane et al. (2005); Daqaq et al. (2006); Bryzek et al. (2003); Vagia et al. (2008)) or even dimensioning purposes. Indeed, due to the very small size of these systems, many parameter values cannot be directly measured and dynamic underlying phenomena are difficult to correctly be described from the only physical analysis; in this case, identification process can be the only way to get reliable models. Several informations and techniques about identification of dynamic systems will be found in (Ljung (1987); Garnier and Young (2004)).

Email addresses: casenave@laas.fr (Céline Casenave), emontseny@laas.fr (Emmanuel Montseny), camon@laas.fr (Henri Camon)

Preprint submitted to Control Engineering Practice
In this paper, parameters, initial conditions and functional components of a dynamic model presenting a dynamic bifurcation are identified. First the differential model of the system, elaborated from physical analysis, is transformed in order to get a new equivalent model, well adapted to continuous time identification method (Garnier and Wang (2008)). The so-obtained model is linear with respect to the parameters to be identified, and continuously depends on noise measurements, what is not the case with the initial form of the model in which derivative operators are involved. Consequently, a simple least-squares resolution can be used to identify the unknown parameters, in such a way that some of the difficulties classically encountered with identification methods, like the non convexity of the cost function for example, are by-passed. Moreover, with such a method, and thanks to the equivalence of the dynamic model and the derived identification one, the identified model remains of continuoustime type, with a clear physical meaning of any of its components, what is not the case when using, for example, black-boxes approaches.

This paper being devoted to practical implementation, the report is mainly formal: some mathematical questions which would have necessitated July 26, 2010 
sophisticated tools of functional analysis are simply mentioned and justified by intuitive physical arguments only.

The paper is organized as follows. In section 2 the physical system and its dynamic model are described. In section 3 a new equivalent formulation devoted to identification is established, and the associated identification problem is given. The solution of this problem is expressed in terms of operatorial pseudo-inversion and the bias reduction method used is presented. Then the numerical resolution of the problem from a discrete set of measurement data is described in section 4 and validated on simulated data in section 5. Finally, the method is implemented on real measurement data and the obtained results are given in section 6 .

\section{The physical system under consideration}

The experimental system is an electrostatically actuated micro-mirror, a view of which ${ }^{1}$ is given in Fig. 1 (the system is in fact made up of four mirrors). The system is composed in two assembled parts. The upper one is a thin plate, the mirror, linked to a thick external rigid frame by two thin and narrow arms, the springs. This part is tailored in the same micro-crystalline silicon layer of a SOI (silicon on insulator) wafer. The lower one comprises a balance-knife-edge with two electrodes distributed on both sides of it. The two parts are assembled in such a manner that the axis of the springs and balance-knife-edge are identical. So the electrodes are located underneath the mirror inducing its rotation (left or right) when a voltage $V$ is applied. The physical limit angle the mirror can reach is denoted $\alpha$, whereas $\theta(t)$ denotes the angle of the mirror at a given time (see Fig. 3).

The development of those micro-mirrors has been conducted with Tronics Microsystems (France), a manufacturer of custom MEMS components. Several configurations of the electrodes can be envisaged, namely the case where electrodes are flat or inclined (cf. Fig. 2 and 3 for flat electrodes configuration).

During the rotation of the mirror, several forces are involved:

- Electrostatic forces : the associated electrostatic moment $M_{e}(\theta, V)$, whose expression depends on the configuration of the system, is

\footnotetext{
${ }^{1}$ The picture is published with courtesy of Tronics Microsystems (France).
}

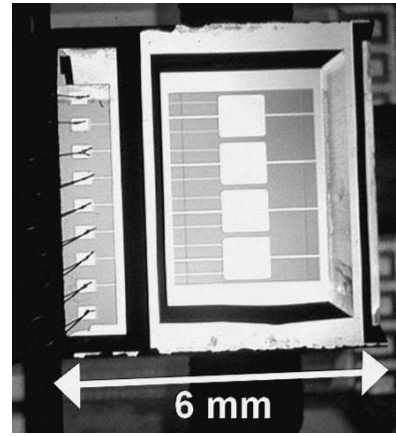

Figure 1: View of the physical system made up of 4 micromirrors.

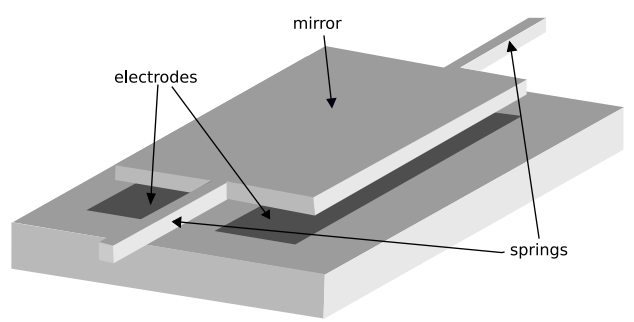

Figure 2: Schematic drawing of the MEMS with flat electrodes

supposed to be of the form (Camon et al. (2008)):

$$
M_{e}(\theta, V)=V^{2} k(\theta) .
$$

- Spring forces: the associated moment is supposed to be proportional to the angle $\theta$, with stiffness constant $K>0$.

- Viscous friction forces : the associated moment $M_{f}(\theta, \dot{\theta})$ is supposed to be of the form:

$$
M_{f}(\theta, \dot{\theta})=-\left(\mu_{0}+v(\theta)\right) \dot{\theta}
$$

with $v(0)=0$.

Remark 1. For simplicity, $\dot{\theta}$ is supposed to remain sufficiently small, in such a way that $v$ only depends on $\theta$. This will be sufficient for the physical problem under consideration in this study. More general nonlinear situations (such as $\left.M_{f}(\theta, \dot{\theta})=-\mu_{0} \dot{\theta}-v(\theta, \dot{\theta})\right)$ could also be treated, up to suitable adaptations involving slightly more sophisticated techniques out of scope in this paper. 


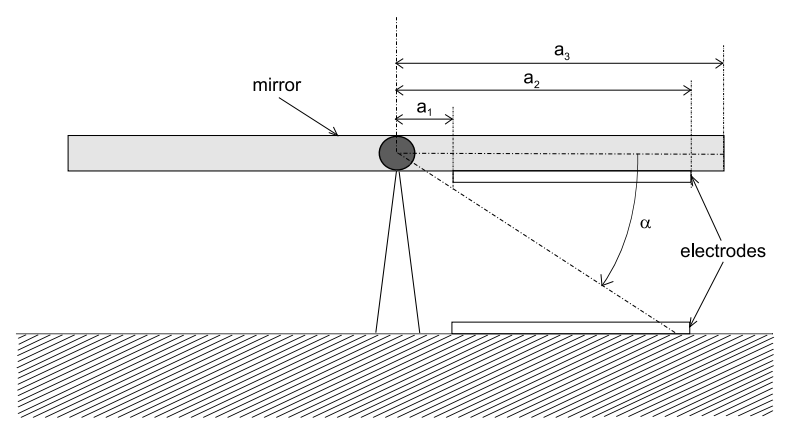

Figure 3: Cross section of the MEMS with flat electrodes

A dynamic model of such a system is obtained by application of the fundamental principle of dynamics:

$$
\begin{aligned}
I \ddot{\theta}+\left(\mu_{0}+v(\theta)\right) \dot{\theta}+K \theta & =V^{2} k(\theta), \\
\text { with the constraint } & : \quad|\theta| \leqslant|\alpha|,
\end{aligned}
$$

where $I$ is the moment of inertia of the system.

Remark 2. In the model, $\theta$ is a negative angle.

The system (3) of input $V$ and output $\theta$, is completed by the initial conditions:

$$
\theta(0)=\theta_{0} \text { and } \dot{\theta}(0)=\theta_{1}
$$

Note that the expression of the terms $v(\theta)$ and $k(\theta)$ will depend on the configuration of the physical system, its geometry, the materials used etc. (see section 6 for a particular example).

The structure of model $(3,4,5)$ proved reliable; it enables to get accurate approximations of the dynamic behavior of the micro-mirror. Nevertheless, due to the fabrication process, essentially the gluing process of the two parts of the mirror, it is realistic to consider that the physical parameters and functions ( $v$ and $k$ ) implied in the model are significantly different from the ideal ones. So, in the sequel, the problem of identification, from real (discrete time) noisy measurement data, of the parameters (for example the moment of inertia), and functions (the electrostatic moment function $k$, etc.) is considered; the initial conditions (5) of model (3) can also be identified. The aim is to get a reliable model of the system with good predictive properties, suitable for example for control purposes such as in Zhu et al. (2007); Liao et al. (2004); Sane et al. (2005); Daqaq et al. (2006); Bryzek et al. (2003).
The identification of the model of such a physical system presents various difficulties frequently encountered in practice:

- The nonlinear dependence of the electrostatic moment on $\theta$ is both ill known and rather singular in the sense that it considerably increases when the angle $\theta$ goes to its maximum value $\alpha$.

- The analysis of this dynamic system reveals the existence of a threshold voltage, called the "pull-in voltage" (Cichalewski et al. (2003)) and denoted $V_{\text {pullin }}^{\text {dyn }}$, below which $\theta$ stabilizes to an angle $\theta_{\text {stab }}(V)$ (see Fig. 9a), whereas beyond this voltage the system becomes unstable and $\theta$ quickly switches to $\alpha$ (see Fig. 9b). In the case of neglected friction moment, this behavior can be highlighted by a phase portrait analysis (cf. Annex A). The existence of such a bifurcation value $V_{\text {pullin }}^{\text {dyn }}$ of the input voltage, which separates two regions with quite different dynamic behaviors, contributes to make the identification problem difficult.

- As often with small size mechanical systems, due to the smallness of the moment of inertia, the dynamic contribution of this term is quite dominated by the viscosity one (that is $|I \ddot{\theta}| \ll$ $\left.\left|\left(\mu_{0}+v(\theta)\right) \dot{\theta}\right|\right)$, except at the very beginning of the motion (when the speed $|\dot{\theta}(t)|$ is very low). Consequently, identification of the moment of inertia from measurement data is a tricky task.

\section{Formulation, analysis and resolution of the identification problem}

In this section is stated and studied the identification problem under consideration, whose aim is to estimate the parameters of model $(3,4,5)$ from measurements of trajectory $\theta$. For mathematical questions, refer for example to Adams and Fournier (2003); Yosida (1980).

\subsection{On the identification technique used}

The aim of system identification is to get a reliable model of the system under consideration from both experimental data and physical knowledge. The identified model can be either discrete (Ljung (1987)) or continuous (Garnier and Young (2004); Young and Garnier (2006); Bingulac and Sinha (1989)). 
method (Garnier and Wang (2008)) is considered, for several reasons:

- The knowledge-model $(3,4,5)$ on which the identification is based is naturally formulated in continuous-time domain.

- The aim is here to get a reliable continuoustime model of the system, with the same structure as model $(3,4,5)$, in order to validate the modelling. Continuous-time identification methods directly provide such a model, which then can be used for simulation, prediction or control purposes.

- Here, the identification process is also used to estimate the physical parameters and characteristic functions of the system. In such cases, identification methods based on a continuoustime model are often used, because the obtained estimates are strongly linked to the physical parameters of the system. In other words, the identified model has a clear physical meaning, what is not the case with traditional discrete-time identification methods (Ljung (1987)).

The knowledge-model $(3,4,5)$ has the particularity to be linear with respect to parameters $I, \mu_{0}$, $K$, and functions $k$ and $v$. To take advantage to this linearity, an identification method based on the minimization of the equation error (Mahata and Garnier (2006); Garnier and Wang (2008)) will be used. To apply such a method, the model has first to be transformed by means of suitable global operations, in such a way that the new equivalent model formulation is specifically adapted to the identification problem, thanks to the following essential properties: first, the linearity with respect to the parameters to be identified is preserved, and second, the continuous dependence on noise measurements is restored, this last property being impossible to get in the initial form of the model because of the presence of derivative operators.

\subsection{Transformation of the model for identification purposes}

\subsubsection{Electrostatic and viscous friction moments}

As said previously, the expression of electrostatic and viscous friction moments depends on the physical system. Even if in some ideal cases their expression can be evaluated (see Appendix A), in general they may not be accurately known, and it can be interesting to also identify the functions $k$ and $v$. In this paper, $k$ and $v$ are identified under the form:

$$
\begin{aligned}
k(\theta) & =\sum_{l} c_{l} \mathbf{k}_{l}(\theta), \\
v(\theta) & =\sum_{q} \mu_{q} \mathbf{v}_{q}(\theta),
\end{aligned}
$$

where the basis functions $\mathbf{k}_{l}$ and $\mathbf{v}_{q}$ are all known; the parameters to be identified are then the real coefficients $c_{l}$ and $\mu_{q}$. Concretely, $\mathbf{k}_{l}$ and $\mathbf{v}_{q}$ are chosen in such a manner that $k$ and $v$ can be approximated by finite sums with few terms. From the theoretical point of view, these sums can be finite or not and implicitly refer to functional Hilbert spaces generated by $\mathbf{k}_{l}$ and $\mathbf{v}_{q}$ (supposed orthonormal for simplicity), to which belong $k$ and $v$.

In the sequel, the following notations are used:

$$
\begin{aligned}
c & :=\left(c_{1}, c_{2}, \ldots\right)^{T} \in \ell^{2}, \\
\mathbf{k}(\theta) & :=\left(\mathbf{k}_{1}(\theta), \mathbf{k}_{2}(\theta), \ldots\right), \\
\mu & :=\left(\mu_{1}, \mu_{2}, \ldots\right)^{T} \in \ell^{2}, \\
\mathbf{v}(\theta) & :=\left(\mathbf{v}_{1}(\theta), \mathbf{v}_{2}(\theta), \ldots\right),
\end{aligned}
$$

and

$$
\mathbf{w}(\theta):=\left(\mathbf{w}_{1}(\theta), \mathbf{w}_{2}(\theta), \ldots\right)
$$

where $\mathbf{w}_{i}$ is the antiderivative of $\mathbf{v}_{i}$ such that $\mathbf{w}_{i}(0)=0$. Then, it can be written under a condensed form:

$$
\begin{aligned}
& k(\theta)=\mathbf{k}(\theta) c, \\
& v(\theta)=\mathbf{v}(\theta) \mu .
\end{aligned}
$$

\subsubsection{Initial conditions}

The system (3), defined on $t>0$ and with initial conditions (5), can be equivalently replaced by the following one defined on $t \in \mathbb{R}$ with $\left.\theta\right|_{\mathbb{R}^{-}}=0$ (see Annex B):

$$
I \partial_{t}^{2} \theta+\mu_{0} \partial_{t} \theta+\partial_{t} \mathbf{w}(\theta) \mu+K \theta=V^{2} \mathbf{k}(\theta) c+a \delta+b \delta^{\prime},
$$

where the coefficients $a$ and $b$ are biunivocally linked with initial conditions $\theta_{0}$ and $\theta_{1}$. The interest of this formulation lies in the fact that $a$ and $b$ are linearly involved, and so initial conditions can be easily deduced from the estimates of $a$ and $b$ if necessary.

Remark 3. The time derivative operator in the sense of distributions is denoted $\partial_{t}$, or simply $(.)^{\prime}$. Its right-inverse is defined on any function $u$ with 
support in $\left[t_{0},+\infty\left[\right.\right.$ (that is $u(t)=0 \forall t<t_{0}$ ) by $u \mapsto \int_{-\infty}^{t} u(\tau) d \tau$ and is denoted $\partial_{t}^{-1}$. The notation $\dot{u}$ designates the derivative of $u$ in the sense of functions. In particular, for a function $u$ continuous except at $t=0$ with right and left limits at 0 (Yosida (1980)):

$$
\partial_{t} u=\dot{u}+\left(u\left(0^{+}\right)-u\left(0^{-}\right)\right) \delta,
$$

where $\delta$ designates the Dirac impulse.

\subsubsection{Prefiltering with an invertible convolution operator}

Under constraint (4), model (15) can be transformed by composition with any causal convolution operator $\mathcal{H}:=H\left(\partial_{t}\right)$ with impulse response $h=\mathcal{L}^{-1} H$, in order to get an equivalent equation well adapted to identification problems:

$$
\begin{aligned}
\left(\mathcal{H} \circ \partial_{t}^{2}\right) & \theta I+\left(\mathcal{H} \circ \partial_{t}\right) \theta \mu_{0}+\mathcal{H}\left(\partial_{t} \mathbf{w}(\theta)\right) \mu \\
& +(\mathcal{H} \theta) K=V^{2} \mathcal{H}(\mathbf{k}(\theta)) c+h a+h^{\prime} b .
\end{aligned}
$$

Such a transformation is indeed often used to mitigate the problem of ill-conditioning of the computation of derivatives from sampled noisy data (Young (1965b); Mahata and Garnier (2006)) (see paragraph 3.4).

\subsubsection{Case of multiple trajectories}

Consider a set of trajectories $\theta^{j}, j=1: J$ obtained with input voltages $V_{j}$. The associated initial conditions are denoted $\theta_{0}^{j}$ and $\theta_{1}^{j}$, and the coefficients defined in section 3.2.2 are denoted $a^{j}$ and $b^{j}$. Then, without any change of notations, model (17) can be extended to the general case of multiple trajectories simply by defining:

$$
\begin{gathered}
\theta=\left(\theta^{1}, \ldots, \theta^{J}\right)^{T}, \\
\theta_{0}=\left(\theta_{0}^{1}, \ldots, \theta_{0}^{J}\right)^{T}, \theta_{1}=\left(\theta_{1}^{1}, \ldots, \theta_{1}^{J}\right)^{T}, \\
a=\left(a^{1}, \ldots, a^{J}\right)^{T}, \quad b=\left(b^{1}, \ldots, b^{J}\right)^{T}, \\
\mathbf{w}(\theta)=\left[\begin{array}{ccc}
\mathbf{w}_{1}\left(\theta^{1}\right) & \mathbf{w}_{2}\left(\theta^{1}\right) & \ldots \\
\vdots & \vdots & \\
\mathbf{w}_{1}\left(\theta^{J}\right) & \mathbf{w}_{2}\left(\theta^{J}\right) & \ldots
\end{array}\right], \\
\mathbf{k}(\theta)=\left[\begin{array}{ccc}
\mathbf{k}_{1}\left(\theta^{1}\right) & \mathbf{k}_{2}\left(\theta^{1}\right) & \ldots \\
\vdots & \vdots & \\
\mathbf{k}_{1}\left(\theta^{J}\right) & \mathbf{k}_{2}\left(\theta^{J}\right) & \ldots
\end{array}\right], \\
\text { and } V=\left[\begin{array}{ccc}
V_{1} & & 0 \\
& \ddots & \\
0 & & V_{J}
\end{array}\right] .
\end{gathered}
$$

\subsubsection{Synthetic formulation}

In model (17), parameters are linearly involved and can be identified up to a multiplicative constant (model with one degree of freedom): it is necessary to know one of the parameters to be able to fix the other ones. So in the sequel, the coefficients to be identified will be $I, \mu, K, c$ (and possibly $a, b$ for initial conditions) while, for simplicity:

$$
\mu_{0}=1
$$

The vector:

$$
\lambda:=\left(I, \mu, K, c, a^{T}, b^{T}\right)^{T} \in \mathcal{E}
$$

with $\mathcal{E}:=\mathbb{R} \times \ell^{2} \times \mathbb{R} \times \ell^{2} \times \mathbb{R}^{2 J}$, is the vector of parameters to be identified. The exact (but unknown) value of $\lambda$ will be denoted $\lambda_{0}$.

For convenience, the following operators will be used:

$$
\mathcal{H}_{1}:=\mathcal{H} \circ \partial_{t} \text { and } \mathcal{H}_{2}:=\mathcal{H} \circ \partial_{t}^{2}
$$

Thus, the vector operator $\mathcal{A}_{\theta}: \mathcal{E} \longrightarrow L^{2}\left(0, T ; \mathbb{R}^{J}\right)$ will be defined by:

$\mathcal{A}_{\theta}=\left[\begin{array}{llllll}\mathcal{H}_{2} \theta & \mathcal{H}_{1}(\mathbf{w}(\theta)) & \mathcal{H} \theta & -V^{2} \mathcal{H}(\mathbf{k}(\theta)) & -h & -h^{\prime}\end{array}\right]$

and:

$$
b_{\theta}:=-\mathcal{H}_{1} \theta \in L^{2}\left(0, T ; \mathbb{R}^{J}\right) .
$$

So under constraint (4), model (17) can be equivalently written under the linear regression form:

$$
\mathcal{A}_{\theta} \lambda=b_{\theta} .
$$

Remark 4. Note that obviously:

$$
A_{\theta} \lambda_{0}=b_{\theta}
$$

Remark 5. For simplicity, all the trajectories $\theta^{j}$ are defined on the same $[0, T]$. For a more general formulation, simply take $\Pi_{j=1}^{J} L^{2}\left(0, T^{j}\right)$ instead of $L^{2}\left(0, T ; \mathbb{R}^{J}\right)$.

\subsection{Formulation and resolution of the identifica- tion problem}

The problem is to estimate $\lambda$ from experimental data of the form:

$$
\theta_{\mathrm{m}}=\theta+\eta
$$

with $\eta$ some additive measurement noise. 
Such a problem can be expressed as the following ordinary least squares problem:

$$
\min _{\lambda \in \mathcal{E}}\left\|\mathcal{A}_{\theta_{\mathrm{m}}} \lambda-b_{\theta_{\mathrm{m}}}\right\|_{L^{2}\left(0, T ; \mathbb{R}^{J}\right)}^{2},
$$

whose solution $\lambda^{*}$ is classically given by:

$$
\lambda^{*}=\mathcal{A}_{\theta_{\mathrm{m}}}^{\dagger} b_{\theta_{\mathrm{m}}},
$$

where $\mathcal{A}_{\theta_{\mathrm{m}}}^{\dagger}$ designates the pseudo-inverse of operator $\mathcal{A}_{\theta_{\mathrm{m}}}$, defined by (Ben-Israel and Greville (2003)):

$$
\mathcal{A}_{\theta_{\mathrm{m}}}^{\dagger}=\left(\mathcal{A}_{\theta_{\mathrm{m}}}^{*} \mathcal{A}_{\theta_{\mathrm{m}}}\right)^{-1} \mathcal{A}_{\theta_{\mathrm{m}}}^{*} .
$$

The statistical properties of estimator $\lambda^{*}$ are well known (Ljung (1987)):

- When the data are noise-free, the estimator $\lambda^{*}$ is exact (thanks to (25)):

$$
A_{\theta}^{\dagger} b_{\theta}=\lambda_{0} .
$$

- When the data are corrupted by additive measurement noise, the estimator $\lambda^{*}$ is biased (Ljung (1987)) because the regressor $\mathcal{A}_{\theta_{\mathrm{m}}}$ depends on the measurement noise. In this paper, a method of bias estimation is proposed and described in paragraph 3.5. Several others methods can be found for example in (Mahata and Garnier (2006); Young (1970); Söderström and Mahata (2002); Welsh et al. (2007); Mahata and Garnier (2005)).

\subsection{On the prefiltering operator $\mathcal{H}$}

The numerical computation of estimator $\lambda^{*}$ necessitates to compute estimations of $\mathcal{A}_{\theta_{\mathrm{m}}}$ and $b_{\theta_{\mathrm{m}}}$ from sampled noisy data. Because some derivatives are involved in model (3), the composition by a suitable convolution operator $\mathcal{H}$ is in general necessary to identify the system. Different kind of filters can be considered (Young (1965a); Saha et al. (1982); Sagara and Zhao (1990); Jemni and Trigeassou (1996)). In this paper, a state variable filter (Young (1965b); Mahata and Garnier (2006); Garnier and Wang (2008)), namely (in this case) a second order filter (because the maximal degree of derivatives involved in model (17) is two) obtained by composition of identical first order filters, is used:

$$
H(p)=\frac{\sigma^{2}}{(p+\sigma)^{2}},
$$

with $\sigma>0$ the cutoff frequency. With such a filtering, high frequencies are attenuated $\left(|H(i \omega)|^{2} \underset{H . F}{\sim}\right.$ $\left.\frac{1}{\omega^{2}}\right)$, without low ones being amplified $\left(|H(i \omega)|^{2} \underset{\text { L.F }}{\stackrel{\text { r.F }}{\sim}}\right.$ $1)$ : thus, the measurement noise is not amplified by terms $\left(\mathcal{H} \circ \partial_{t}\right) \theta,\left(\mathcal{H} \circ \partial_{t}^{2}\right) \theta$ and $\mathcal{H}\left(\partial_{t} \mathbf{w}(\theta)\right)$ in model (17).

In practice, the cutoff frequency $\sigma>0$ of the filter is chosen in such a way that $\left\|\mathcal{A}_{\theta_{\mathrm{m}}} \lambda-b_{\theta_{\mathrm{m}}}\right\|^{2}$ is as "small" as possible. This value, or even the transfer function $H(p)$ could also be optimized in order to minimize the equation error.

Remark 6. If $H\left(\partial_{t}\right)$ is the filter defined by (31), then:

$$
\begin{gathered}
\left(H\left(\partial_{t}\right) u\right)(t)=\sigma^{2} \int_{0}^{t}(t-s) e^{-\sigma(t-s)} u(s) d s, \\
h(t)=\sigma^{2} t e^{-\sigma t}, h^{\prime}(t)=\sigma^{2}(1-\sigma t) e^{-\sigma t} .
\end{gathered}
$$

\subsection{Bias reduction}

As said previously, the estimator $\lambda^{*}$ is biased in presence of noise. A simple empirical methodology (useful when the measurement noise is small enough) is presented here to improve the identification accuracy by estimating the bias of $\lambda^{*}$. For that, the following hypothesis are made:

1) the bias depends continuously on the parameters to be identified;

2) in the identification error $\lambda_{0}-\lambda^{*}$, the bias term $\varepsilon_{\lambda^{*}}$ is dominant;

3) the bias $\varepsilon_{\lambda^{*}}$ has a small variance compared to the one of the identification error $\lambda_{0}-\lambda^{*}$; in other words, the bias is little dependent on the particular trajectory of the measurement noise (it mainly depends on the noise statistical characteristics).

Hypothesis 1) is physically reasonable and in fact necessary to get robust identification. Hypothesis 2) can appear rather questionable; but note that if it is not satisfied, the process described here-after will be neutral: no improvement will be obtained, and the identification error will not significantly be increased. Finally, hypothesis 3) involves some subtle underlying ergodic properties, in general delicate to state, but which are most of time satisfied in practice. 
Based on the above properties, a simple method to estimate the identification bias (and then subtract it from the identified parameter $\left.\lambda^{*}\right)$ is proposed:

- First the identification process is applied on the available experimental measured data. Thanks to hypothesis 2 ), the relation between the exact parameter $\lambda_{0}$, the identified one $\lambda^{*}$ and the associated bias $\varepsilon_{\lambda^{*}}$ can be reduced to:

$$
\lambda^{*} \simeq \lambda_{0}+\varepsilon_{\lambda^{*}}
$$

- Then consider a set of data numerically simulated from the identified model (defined by $\lambda^{*}$ ), with the same noise level as the measured ones (on which the identification process is implemented). The parameters are identified from this simulated set of data: the new identified vector of parameters is denoted by $\lambda_{1}$.

- Now, thanks to hypothesis 2), the following estimation of the bias of $\lambda_{1}$ (in fact the opposite of identification error) can be considered:

$$
\varepsilon_{\lambda_{1}}^{*}:=\lambda_{1}-\lambda^{*}
$$

which, thanks to hypotheses 1) and 3), is supposed to be close to the unknown value $\varepsilon_{\lambda^{*}}$ if the noise level is small enough.

- Then, the identification of vector $\lambda$ is expected to be improved by considering the following estimator of $\lambda$ :

$$
\lambda_{1}^{*}:=\lambda^{*}-\varepsilon_{\lambda_{1}}^{*}=2 \lambda^{*}-\lambda_{1} .
$$

This process can possibly be iterated from the new value $\lambda_{1}^{*}$, and so on until $\varepsilon_{\lambda_{n}}$ stabilizes around 0 .

Such a bias reduction will be implemented on simulated data in order to highlight its efficiency (see paragraph 5.3).

Remark 7. Because mathematical models used for identification in general cannot describe all the complex phenomena involved in physical situations, in many cases, the bias error can be drowned in larger errors resulting from such "structural ${ }^{2}$ " model imperfections. In such cases, bias reduction is irrelevant.

\footnotetext{
${ }^{2}$ Note that when the model presents some "structural imperfection", $\lambda^{*} \neq \lambda_{0}$ even for noise-free measurements.
}

\section{Numerical Formulation}

In the previous section, the continuous time (infinite dimensional) identification problem has been introduced. Now, its numerical resolution is tackled.

\subsection{Numerical resolution of (27)}

First of all, if (13) and (14) are not finite sums, they must be replaced, for numerical implementation, by truncations at finite orders $L$ and $Q$ :

$$
\begin{aligned}
\mathbf{k}\left(\theta^{j}\right) c & \simeq \sum_{l=1}^{L} c_{l} \mathbf{k}_{l}\left(\theta^{j}\right) \\
\mathbf{v}\left(\theta^{j}\right) \mu & \simeq \sum_{q=1}^{Q} \mu_{q} \mathbf{v}_{q}\left(\theta^{j}\right) .
\end{aligned}
$$

The numbers $L$ and $Q$ must be chosen according to the best compromise between errors generated by truncation and errors generated by the presence of measurement noise, or even by some structural lacks of the model (which cannot describe the physical system in its whole complexity). In practice, such a choice is achieved empirically.

So, $\mathbf{k}(\theta)$ and $\mathbf{w}(\theta)$ will now designate the respective finitedimensional matrices:

$$
\begin{array}{r}
{\left[\begin{array}{ccc}
\mathbf{w}_{1}\left(\theta^{1}\right) & \cdots & \mathbf{w}_{Q}\left(\theta^{1}\right) \\
\vdots & & \vdots \\
\mathbf{w}_{1}\left(\theta^{J}\right) & \cdots & \mathbf{w}_{Q}\left(\theta^{J}\right)
\end{array}\right]} \\
\text { and }\left[\begin{array}{ccc}
\mathbf{k}_{1}\left(\theta^{1}\right) & \cdots & \mathbf{k}_{L}\left(\theta^{1}\right) \\
\vdots & & \vdots \\
\mathbf{k}_{1}\left(\theta^{J}\right) & \cdots & \mathbf{k}_{L}\left(\theta^{J}\right)
\end{array}\right] .
\end{array}
$$

The problem (27) is then discretized. Let $\left\{t_{n}\right\}_{n=1: N}$ be a time discretization ${ }^{3}$. For any $t_{n}$ :

$$
\left(\mathcal{A}_{\theta} \lambda\right)\left(t_{n}\right)=b_{\theta}\left(t_{n}\right)
$$

Let $E:=\mathbb{R} \times \mathbb{R}^{Q} \times \mathbb{R} \times \mathbb{R}^{L} \times\left(\mathbb{R}^{J}\right)^{2}$ and $\mathrm{A}_{\mathrm{m}}: E \longrightarrow$ $\left(\mathbb{R}^{J}\right)^{N}$ be the block-matrix:

$$
\mathrm{A}_{\mathrm{m}}:=\left[\begin{array}{c}
\mathrm{A}_{\theta_{m}}\left(t_{1}\right) \\
\vdots \\
\mathrm{A}_{\theta_{m}}\left(t_{N}\right)
\end{array}\right],
$$

\footnotetext{
${ }^{3}$ Here again, the same $N$ has been chosen for simplicity. Possible different $N^{j}$ associated with $\theta^{j}$ can be considered with ad-hoc adaptations.
} 
where $\mathrm{A}_{\theta_{\mathrm{m}}}\left(t_{n}\right): E \longrightarrow \mathbb{R}^{J}$ is the matrix defined by:

$$
\begin{aligned}
& \mathrm{A}_{\theta_{\mathrm{m}}}\left(t_{n}\right):= \\
& {\left[\begin{array}{llll}
\left(\mathcal{H}_{2} \theta_{\mathrm{m}}\right)\left(t_{n}\right) & \left(\mathcal{H}_{1}\left(\mathbf{w}\left(\theta_{\mathrm{m}}\right)\right)\right)\left(t_{n}\right) & \left(\mathcal{H} \theta_{\mathrm{m}}\right)\left(t_{n}\right) \\
\quad-V^{2}\left(t_{n}\right) & \left(\mathcal{H}\left(\mathbf{k}\left(\theta_{\mathrm{m}}\right)\right)\right)\left(t_{n}\right) & -h\left(t_{n}\right) & -h^{\prime}\left(t_{n}\right)
\end{array}\right] .}
\end{aligned}
$$

Let also define:

$$
\mathrm{b}_{\mathrm{m}}:=\left[\begin{array}{c}
-\left(\mathcal{H}_{1} \theta_{\mathrm{m}}\right)\left(t_{1}\right) \\
\vdots \\
-\left(\mathcal{H}_{1} \theta_{\mathrm{m}}\right)\left(t_{N}\right)
\end{array}\right] \in\left(\mathbb{R}^{J}\right)^{N} .
$$

Remark 8. The computation of the components of $A_{m}$ and $b_{m}$ is detailed in Appendix $C$ in the case where a continuous-time equivalent of $\theta_{\mathrm{m}}$ is derived from discrete data by means of cubic spline interpolation (see section 4.2).

The identification problem (27) is then approximated by the following discrete-one (obtained by application of the rectangles method to the integral which defines the norm $\left.\|\cdot\|_{L^{2}\left(0, T ; \mathbb{R}^{J}\right)}^{2}\right)$ :

$$
\min _{\lambda \in E}\left\|\mathrm{~A}_{\mathrm{m}} \lambda-\mathrm{b}_{\mathrm{m}}\right\|_{\left(\mathbb{R}^{J}\right)^{N}}^{2},
$$

where $\|c\|_{\left(\mathbb{R}^{J}\right)^{N}}^{2}=\sum_{i=1}^{N \times J}\left|c_{i}\right|^{2}$ (here the time-step $\Delta t$ is supposed to be constant). Its solution is classically given by:

$$
\lambda^{*}=\left(\mathrm{A}_{\mathrm{m}}^{T} \mathrm{~A}_{\mathrm{m}}\right)^{-1} \mathrm{~A}_{\mathrm{m}}^{T} \mathrm{~b}_{\mathrm{m}} .
$$

Remark 9. The dimension of the square matrix $\mathrm{A}_{\mathrm{m}}^{T} \mathrm{~A}_{\mathrm{m}}$ is rather small (equals to the number of coefficients to be identified).

Remark 10. If the matrix $\mathrm{A}_{\mathrm{m}}^{T} \mathrm{~A}_{\mathrm{m}}$ is illconditioned, standard methods as preconditioning matrix or penalization parameter can be used (see section 5).

\subsection{Discrete data interpolation}

In practice, for any $j=1: J$, a discrete set of data $\left\{\theta_{\mathrm{m}}^{j, k}\right\}_{k=1: K}$ is available, where $\theta_{\mathrm{m}}^{j, k}$ is a measurement (with possible additive noise) of $\theta^{j}\left(t_{k}\right)=$ $\theta\left(t_{k}, V_{j}\right)$ with $t_{k+1}=t_{k}+\Delta t$. The computation of the components of $\mathrm{A}_{\mathrm{m}}$ and $\mathrm{b}_{\mathrm{m}}$ from these discrete data is important: depending on the way computations are made, an error or a deterministic bias can be generated. To avoid this bias, the filter $\mathcal{H}$ has to be simulated by means of the exponential matrix method (see Sinha and Rao (1991) and Appendix
C), which depends on the assumption made on the inter-sample behavior of the signals. Most often, the sampled signal is assumed to remain constant or to vary linearly between the sampling instants. In this paper, a cubic spline interpolation of the set of data is considered: as cubic splines are both regular (twice differentiable) and optimal in the sense of a quadratic functional (see Schumaker (2007) for details), it makes them robust and efficient for interpolation. So between the sampling instants, the signals are assumed to be polynomial functions of degree 3. The computation of the components of $A_{m}$ and $b_{m}$ with such an assumption is detailed in Appendix C.

Denote $\theta_{\mathrm{m}}^{j}: t \mapsto \theta_{\mathrm{m}}^{j}(t)$ the cubic spline interpolation of the set $\left\{\left(t_{k}, \theta_{\mathrm{m}}^{j, k}\right)\right\}_{k}$. Then, the so-obtained interpolated measured trajectories can be written:

$$
\theta_{\mathrm{m}}^{j}=\theta^{j}+\eta_{\mathrm{m}}+\eta_{\mathrm{i}},
$$

where $\theta^{j}$ is the exact (unknown) trajectory solution of (17) (with $\left.V=V_{j}\right), \eta_{\mathrm{m}}$ is the (interpolated) measurement noise and $\eta_{\mathrm{i}}$ is an additional noise resulting from interpolation errors. In the sequel the following notation will be used:

$$
\eta=\eta_{\mathrm{m}}+\eta_{\mathrm{i}}
$$

In the same way, spline interpolations of the sets $\left\{\left(t_{k}, \mathbf{w}_{q}\left(\theta_{\mathrm{m}}^{j, k}\right)\right)\right\}_{k}$, and $\left\{\left(t_{k}, \mathbf{k}_{l}\left(\theta_{\mathrm{m}}^{j, k}\right)\right)\right\}_{k}$ are considered. Then, it follows a continuous-time identification problem of the form (27), whose numerical solution is obtained as described in the previous section.

Remark 11. The discretization introduced in the previous section can be different from the set $\left\{t_{k}\right\}_{k=1: K}$ of measurement times.

\section{Application to simulated data}

In this section, the method previously described is implemented on data built from numerical simulations of model (3). The coefficients and functions have been chosen in the same order of magnitude than the ones of the experimental system studied in section 6 .

The system of model (3) is considered, with ${ }^{4}$ :

$$
\begin{aligned}
& k(\theta)=\sum_{l=1}^{6} c_{l} \mathbf{k}_{l}(\theta) \quad \text { where } \mathbf{k}_{l}(\theta)=-\theta^{l-1}, \\
& v(\theta)=\mu_{1} \theta^{3},
\end{aligned}
$$

\footnotetext{
${ }^{4}$ These choices will be explained in section 6 .
} 
and the following parameters (normalized values, i.e. $\left.\mu_{0}=1\right)$ :

$$
\begin{aligned}
& I=3.7 \times 10^{-5}, \\
& \mu_{1}=-2.0 \times 10^{5} \\
& K=3.6 \times 10^{3}, \\
& c=\left(0.02,-0.7,304,2.810^{4}, 2.210^{6}, 3.610^{7}\right)^{T} .
\end{aligned}
$$

The identification method is implemented on sets of measured data derived from two simulated ${ }^{5}$ trajectories $\theta^{j}, j=1,2$ with $\theta_{0}^{j}=0, \theta_{1}^{j}=0$ and $V \in\{27.4,28.0\}$ (see Fig. 4); note that $27.4<$ $V_{\text {pullin }}^{\text {dyn }}<28.0$. The discrete measured data are :

$$
\theta_{\mathrm{m}}^{j, k}=\theta^{j}(k \Delta t)+\eta_{k}^{j}
$$

with $\Delta t=2 \times 10^{-5}$ and $\left\{\eta_{k}^{j}\right\}_{k}$ an additive numerical noise.

Remark 12. Here, the initial conditions are not identified, that is the components $a$ and $b$ have been removed from $\lambda$, with ad-hoc adaptations.

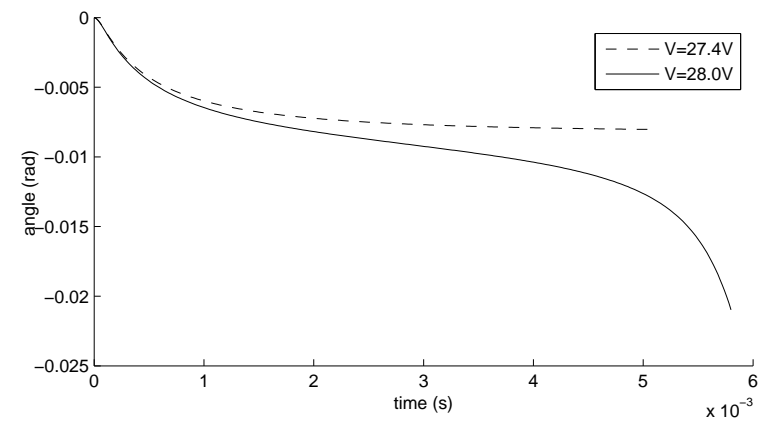

Figure 4: Simulated trajectories $\theta$ used for identification

The identification model is of the form (17) with $v$ and $k$ defined by (49) and the prefiltering operator $\mathcal{H}$ of the form $(31)$ with $\sigma=9.0 \times 10^{3}$; this value has been chosen to be compatible with the problem: $\frac{1}{t_{\max }} \ll \sigma \ll \frac{1}{2 \Delta t}$.

Because some terms of the model are dominated by the others (especially the moment of inertia as explained previously), a preconditioning matrix is necessary to solve the problem. So instead of (45), the following least squares problem is considered:

$$
\min _{\Lambda \in E}\left\|\mathrm{~A}_{\mathrm{m}} D \Lambda-\mathrm{b}_{\mathrm{m}}\right\|_{\left(\mathbb{R}^{J}\right)^{N}}^{2},
$$

\footnotetext{
${ }^{5}$ For simulation, a high precision scheme has been used in such a way that numerical integration errors remain very small.
}

where $\lambda=D \Lambda$, with $D$ a symmetric matrix adapted to the problem. Its solution is given by:

$$
\lambda^{*}=D \Lambda^{*}=D\left(D \mathrm{~A}_{\mathrm{m}}^{T} \mathrm{~A}_{\mathrm{m}} D\right)^{-1} D \mathrm{~A}_{\mathrm{m}}^{T} \mathrm{~b}_{\mathrm{m}} .
$$

The matrix $D$ which was used in practice is given by:

$D=\operatorname{diag}\left(10^{-7}, 10^{-5}, 10^{-2}, 10^{-6}, 10^{-4}, 10^{-2}, 1,1,1\right)$.

These values have been chosen empirically in order to make the matrix $D \mathrm{~A}_{\mathrm{m}}^{T} \mathrm{~A}_{\mathrm{m}} D$ well-conditioned.

In order to globally estimate the identification quality, the following quantity is considered:

$$
\begin{aligned}
\mathrm{E} & =\frac{\sum_{j, k}\left|\theta^{j *}(k \Delta t)-\theta_{\mathrm{m}}^{j, k}\right|}{\sum_{j, k}\left|\theta_{\mathrm{m}}^{j, k}\right|} \\
& \simeq \frac{\sum_{j} \int_{0}^{K \Delta t}\left|\theta^{j}(t)-\theta_{m}(t)\right| d t}{\sum_{j} \int_{0}^{K \Delta t}\left|\theta_{m}(t)\right| d t}
\end{aligned}
$$

it represents the cumulated relative error on all the trajectories used for identification.

\subsection{Identification without measurement noise}

The case where simulated data are noise-free $\left(\eta_{k}=0\right)$ is first considered; the set of measured trajectories is then:

$$
\theta_{\mathrm{m}}=\left\{\theta^{1}(k \Delta t), \theta^{2}(k \Delta t)\right\}
$$

with $V_{1}=27.4, V_{2}=28.0$ (that is $\theta_{\mathrm{m}}^{j}$ are exact solutions of (3) up to numerical simulation errors). In this case, as expected, the results are very good: all the parameters are identified with a maximal relative error less than $0.5 \%$ (note that this identification error is only due to numerical errors). The value of the corresponding quantity $E$ is:

$$
\mathrm{E}=1.815 \times 10^{-5} \text {. }
$$

\subsection{Identification with measurement noise}

Now, data used are:

$$
\begin{aligned}
\theta_{\mathrm{m}} & =\left\{\theta_{\mathrm{m}}^{1, k}, \theta_{\mathrm{m}}^{2, k}\right\} \\
& =\left\{\theta^{1}(k \Delta t)+\eta_{k}^{1}, \theta^{2}(k \Delta t)+\eta_{k}^{2}\right\}
\end{aligned}
$$

with $\left\{\eta_{k}^{j}\right\}_{k}$ some measurement noises of the form $\left\{\varepsilon \eta_{k}^{j}\right\}_{k}$ with $\left\{\eta_{k}^{j}\right\}_{k}$ some unity gaussian white noise sequences and $\varepsilon=10^{-5}$ (such a value has been chosen to get $\left\{\eta_{k}^{j}\right\}_{k}$ comparable with the real data 
case, presented in section 6). Then, the following identified values are obtained:

$$
\begin{aligned}
& I^{*}=3.348 \times 10^{-5}, \\
& \mu_{1}^{*}=-1.113 \times 10^{5}, \\
& K^{*}=3.469 \times 10^{3} ;
\end{aligned}
$$

the identified electrostatic function $k^{*}(\theta)$ is shown in Fig. 5. The value of the corresponding quantity $E$ is in this case:

$$
\mathrm{E}=1.527 \times 10^{-3} .
$$

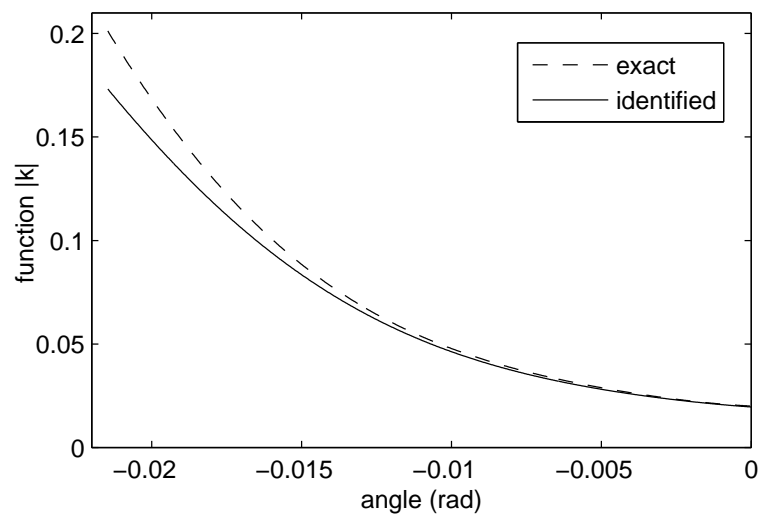

Figure 5: Exact and identified functions $|k|$.

The difference between $\theta^{j}$ and the trajectories $\theta^{j *}$ simulated from the identified model is given in Fig. 6 . In spite of the identification bias, this difference remains small: the identified model closely behaves like the exact one.

In order to highlight the statistical behavior of the estimator $\lambda^{*}$, this identification algorithm has been implemented 12 times with data $\left\{\theta_{\mathrm{m}}^{1, k}, \theta_{\mathrm{m}}^{2, k}\right\}$, $V_{1}=27.4, V_{2}=28.0$, and 12 times with data $\left\{\theta_{m}^{j, k}\right\}_{j=1: 20}, V_{j \leqslant 10}=27.4, V_{j>10}=28.0$, the noise $\left\{\eta_{k}^{j}\right\}_{k}$ being different for each $j$. The dispersion of the collection of estimates in the two cases is given in Fig. 7, where one can see the experimental confidence intervals for the components $I, \mu_{1}$ and $K$ of the estimator $\lambda$. As expected, the bigger the data set, the smaller the intervals.

\subsection{Reduction of identification bias}

First note that the previous results (cf. namely Fig. 7) experimentally validate the hypotheses 2 )

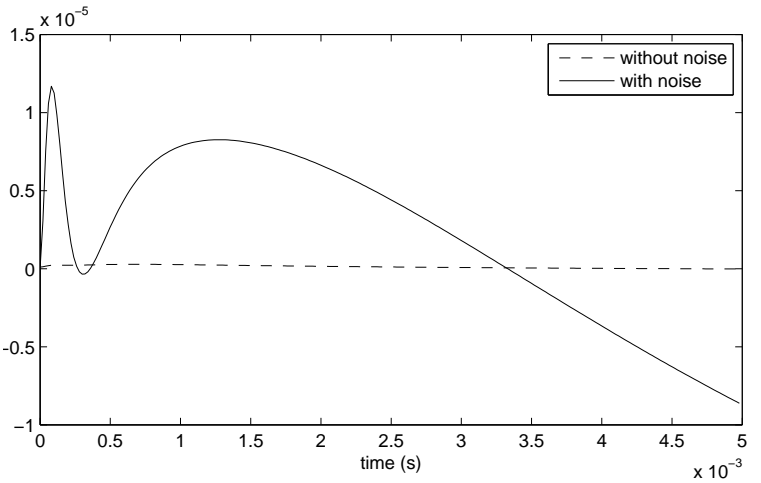

(a) $V=27.4 \mathrm{~V}\left(=V_{1}\right)$

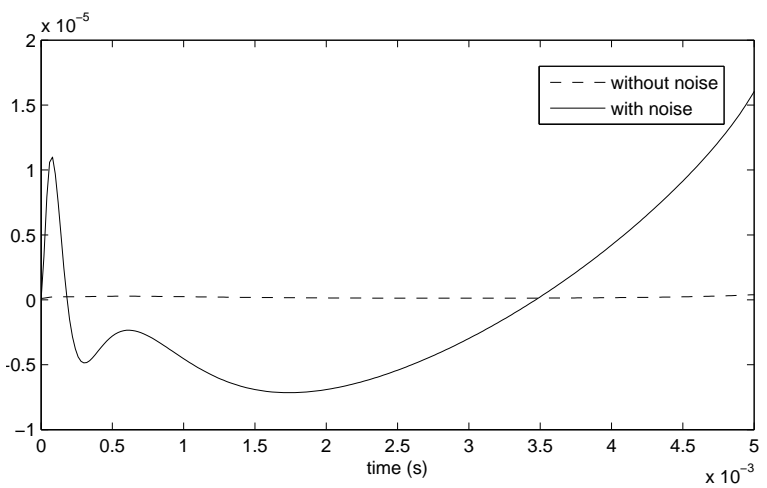

(b) $V=28 \mathrm{~V}\left(=V_{2}\right)$

Figure 6: Error (difference) between $\theta^{j}$ and the trajectories $\theta^{j *}$ simulated from the identified model.

and 3) of section 3.5. Various numerical results (not presented here) with different choices of parameter $\lambda_{0}$ validate hypothesis 1 ) also.

Because the exact value $\lambda_{0}$ is known, an estimate of the identification bias can be determined from (50), (60) and by using the relation (34), in particular for parameters $I, \mu_{1}, K$ :

$$
\begin{aligned}
& \varepsilon_{I^{*}}=-0.35 \times 10^{-5}, \\
& \varepsilon_{\mu_{1}^{*}}=0.887 \times 10^{5}, \\
& \varepsilon_{K^{*}}=-0.131 \times 10^{3},
\end{aligned}
$$

and for function $k$ :

$$
\int_{\alpha}^{0}\left|k(\theta)-k^{*}(\theta)\right| d \theta=1.921 \times 10^{-4} .
$$

Following the process described in section 3.5, a new identification from data simulated with the previously identified $\lambda^{*}$ gives:

$$
\begin{aligned}
& I_{1}=3.080 \times 10^{-5}, \\
& \mu_{1_{1}}=-1.140 \times 10^{5}, \\
& K_{1}=3.326 \times 10^{3},
\end{aligned}
$$




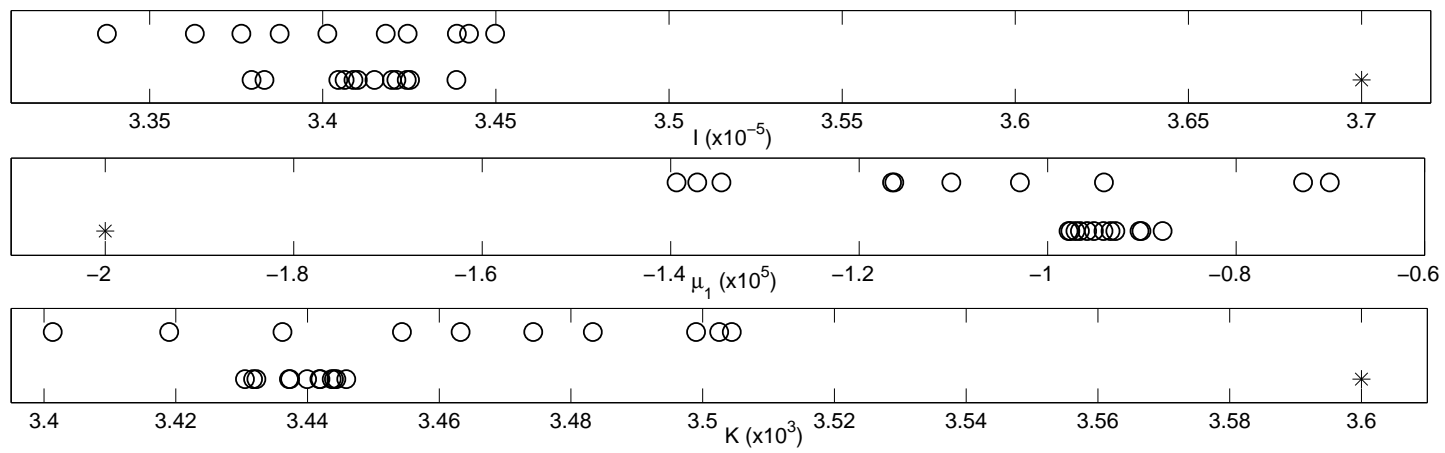

Figure 7: Estimates of $I, \mu_{1}$ and $K$ when the identification is made with 2 or 20 data trajectories (top or bottom of each figure); the $*$ represents the value to be identified.

and the following bias estimates are:

$$
\begin{aligned}
& \varepsilon_{I_{1}}^{*}=-0.268 \times 10^{-5}, \\
& \varepsilon_{\mu_{1_{1}}}^{*}=0.9732 \times 10^{5}, \\
& \varepsilon_{K_{1}}^{*}=-0.1424 \times 10^{3},
\end{aligned}
$$

which, as expected, are clearly close to the values (62). It gives the following new estimates of parameters $I, \mu_{1}, K$ :

$$
\begin{aligned}
& I_{1}^{*}=I^{*}-\varepsilon_{I_{1}}^{*}=3.616 \times 10^{-5}, \\
& \mu_{1_{1}}^{*}=\mu_{1}^{*}-\varepsilon_{\mu_{1}}^{*}=-2.086 \times 10^{5}, \\
& K_{1}^{*}=K^{*}-\varepsilon_{K_{1}}^{*}=3.612 \times 10^{3} .
\end{aligned}
$$

The new estimate of function $k$, denoted $k_{1}^{*}$, is given in Fig. 5. As expected, these estimates are much more precise than $I^{*}, \mu_{1}^{*}, K^{*}$, and $k^{*}$, the error of this improved identification being now (with $\delta \lambda:=$ $\left.\lambda-\lambda_{1}^{*}\right)$ :

$$
\begin{aligned}
\delta I & =0.0839 \times 10^{-5}, \\
\delta \mu_{1} & =-0.0862 \times 10^{5}, \\
\delta K & =0.0118 \times 10^{3}, \\
\int_{\alpha}^{0}\left(k(\theta)-k_{1}^{*}(\theta)\right) d \theta & =1.191 \times 10^{-4},
\end{aligned}
$$

that is more than 10 times smaller than the initial ones (62). Finally, the relative identification errors on the system parameters are:

$$
\begin{aligned}
\left|\frac{\delta I}{I}\right| & =0.0227, \\
\left|\frac{\delta \mu_{1}}{\mu_{1}}\right| & =0.043, \\
\left|\frac{\delta K}{K}\right| & =0.0033, \\
\frac{\int_{\alpha}^{0}\left(k(\theta)-k_{1}^{*}(\theta)\right) d \theta}{\int_{\alpha}^{0} k(\theta) d \theta} & =0.0781 .
\end{aligned}
$$

\section{Application to real measurement data}

In this section, the method is implemented on real data measured on a MEMS like described in section 2. First the physical system and the model used for identification are presented. Then the available measurement data are described. Finally, the obtained results are presented and commented.

\subsection{About the physical system and the model struc- ture}

The MEMS under consideration has flat electrodes as described in Fig. 3, with:

$$
\begin{aligned}
& \alpha=-0.0215 \mathrm{rad}, \\
& a_{1}=150 \mu \mathrm{m}, \\
& a_{2}=280 \mu \mathrm{m}, \\
& a_{3}=300 \mu \mathrm{m} .
\end{aligned}
$$

The width of the mirror is equal to $600 \mu \mathrm{m}$ and its thickness is equal to $10 \mu \mathrm{m}$ (Camon et al. (2008)).

\subsubsection{Electrostatic moment}

A simplified physical analysis of the electrostatic moment leads to the expression: $M_{e}(\theta, V) \propto$ $V^{2} g(\theta)$ with (Camon and Larnaudie (2000)):

$g(\theta)=-\frac{1}{\theta^{2}}\left[\frac{1}{1-\frac{a_{2}}{a_{3}} \frac{\theta}{\alpha}}-\frac{1}{1-\frac{a 1}{a_{3}} \frac{\theta}{\alpha}}+\ln \left(\frac{1-\frac{a_{2}}{a_{3}} \frac{\theta}{\alpha}}{1-\frac{a 1}{a_{3}} \frac{\theta}{\alpha}}\right)\right]$.

If this expression is reliable, then the electrostatic moment can be identified under this form by simply considering (37) with $L=1$ and $\mathbf{k}_{1}=g$. However, due to several approximations in the physical analysis, the function (70) in fact reveals itself ill adapted for large values of $\theta$ (see Fig. 11 and 12). So, the whole electrostatic moment is identified by means of a classical polynomial approximation, that is a function of the form (37) with:

$$
\mathbf{k}_{l}(\theta)=-\theta^{l-1}, l=1: L .
$$


After empirical tests, the polynomial order was chosen equal to:

$$
L=6
$$

it gives a good compromise between the number of parameters and the identification quality.

\subsubsection{Viscosity moment}

A linear viscosity term $\mu_{0} \dot{\theta}$ is not sufficient to correctly describe the viscous moment in its whole, namely when both $|\theta|$ and $|\dot{\theta}|$ become large ${ }^{6}$. So, a nonlinear viscosity term $v(\theta)=\mu_{1} \theta^{3}$ is considered and identified under the form (38) with $Q=1$ and:

$$
\mathbf{v}_{1}(\theta)=\theta^{3} .
$$

\subsubsection{Filtering operator and initial condition pa- rameters}

As explained in section 3.4, the prefiltering operator $\mathcal{H}$ is chosen under the form (31) with $\sigma=$ $9.0 \times 10^{3}$

The expression of parameters $a$ and $b$ (see section 3.2.2) depends on the choice of function $v(\theta)$. In the case where $v(\theta)=\mu_{1} \theta^{3}, a$ and $b$ are given by (see Appendix B):

$$
a=I \theta_{1}+\theta_{0}+\frac{\mu_{1}}{4} \theta_{0}^{4}, \text { and } b=I \theta_{0} .
$$

\subsection{Description of the available measurement data}

Data have been elaborated from $J=36$ measured trajectories of $\theta$, sampled at frequency $50 \mathrm{kHz}$ during $10 \mathrm{~ms}$.

The experimental setup is given in Fig. 8; it is composed of: a laser source, the array of micromirror, a positioning sensor device (PSD), an oscilloscope and a function generator. It is placed on an optical table, and all optical components (laser, MEMS, PSD) are mounted on positioning stages with six degrees of freedom. The function generator is used to generate a periodic square voltage signal applied to a micro-mirror with a frequency low enough to capture the complete response of the mechanical structure. The PSD is composed of four diodes (two for the $\mathrm{X}$ axis and two for the $\mathrm{Y}$ axis) allowing to measure in both perpendicular direction. At the point of impact, four photocurrents are generated and the position is determined by the

\footnotetext{
${ }^{6}$ The physical reasons of such a behavior are not yet quite clear. A possible explanation is that when $\theta$ becomes close to $\alpha$ (see Fig. 3), flow of air compressed between the two electrodes can widely slow down the movement.
}

ratio of the difference by the sum of these currents. All measurements are carried out in dark to avoid parasitic effects of ambient light in the room. The surface of the PSD used is equal to $4 \mathrm{~cm}^{2}$. It is positioned in order to ensure a normal incidence at the middle of the mechanical trajectory and at the maximum distance to have the maximum precision on the location of the impact point. The value of angle $\theta$ is deduced from simple trigonometric transformation of the $\mathrm{X}$ and $\mathrm{Y}$ coordinates of the impact point. On the oscilloscope, the voltage step applied to the micro-mirror and the sensors signal in the $\mathrm{X}$ and $\mathrm{Y}$ directions are observed in the same window. Data are stored in the oscilloscope with 105 points for each channel during a period.

Finally, 36 sets of data $\left\{\theta_{\mathrm{m}}^{j, k}\right\}_{k=1: K}$ with $K=501$ are available. The associated input voltages are described here-after. During the first $5 \mathrm{~ms}$, the voltage between electrodes is fixed at a constant value $V_{j}$; thus, the system either switches or stabilizes at $\theta_{\infty}^{j}<\alpha$. During the next $5 \mathrm{~ms}$, the two electrodes are at the same potential: the system then asymptotically returns to its rest position (say $\theta=0, \dot{\theta}=0)$. The 36 voltage values $V_{j}$ are distributed from $6.085 \mathrm{~V}$ to $97.36 \mathrm{~V}$, with $V_{j}<V_{j+1}$.

Two examples of data trajectories are given in Fig. 9a and 9b. Note, in Fig. 9b, the saturation zone $[0.0045 \mathrm{~s}, 0.005 \mathrm{~s}]$ where $\theta=\alpha$. For identification, the corresponding points are simply suppressed because they are not compatible with model (24) (which cannot describe this saturation phenomenon). In the same way, only dynamic parts of data are used for identification: for example, the zone $[0.0015 \mathrm{~s}, 0.005 \mathrm{~s}]$ in Fig. 9a has not been taken into account because poorly representative of $d y-$ namic behaviors. Finally, because of the probable presence of residual electrostatic charges not taken into account in the model, the data of the return phase obtained after a switch to $\alpha$ (that is, for example, the zone $[0.005 \mathrm{~s}, 0.01 \mathrm{~s}]$ in Fig. $9 \mathrm{~b}$ ) are not used for identification.

Then, the sets of data effectively used for identification are of the form $\left\{\theta_{\mathrm{m}}^{j, k}\right\}_{k=1: K_{j}}$, with $K_{j}$ varying following the part of the trajectory compatible with model (24).

\subsection{Identification results}

The model is identified following two different ways. A first possibility is to identify all the parameters as it has been presented in section 4 . Another one consists in using the fact that, thanks to 


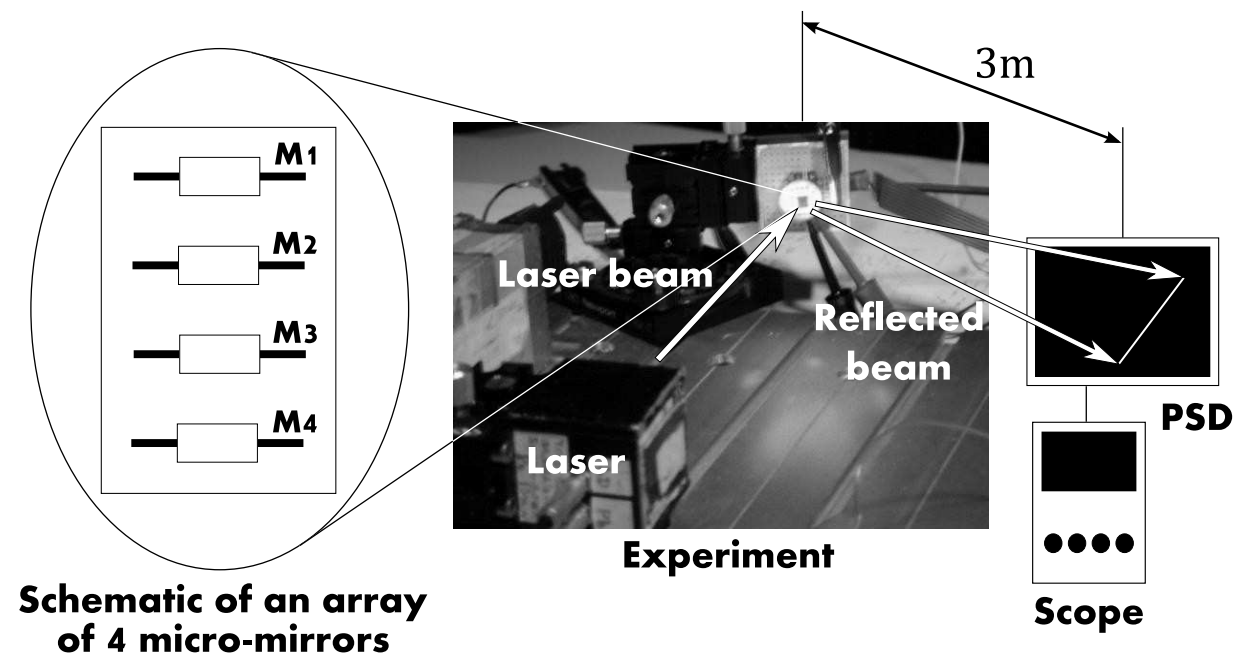

Figure 8: Experimental setup

the particular form of $V_{j}(t)$ (constant first and then switching to 0 ) the identification process can be split into two steps: in the first one (return to rest of the mirror), only the parameters $I, K, a, b$ are identified, while in the second one (stabilization/switch of the mirror) $\mu, c$ are identified with the previous parameters considered as known (see paragraph 6.3.2 for detailed explanation). As shown here after, both identifications give quite similar results.

In both cases, the same preconditioning matrix $D$ as the one used with simulated data (see section 5) is considered.

As in the case of simulated data, bias reduction can also be relevant. As the structure of the model considered for identification is reliable ${ }^{7}$, and because the noise level added to simulated data (see paragraph 5) is similar to the one of real measurement data, the context of identification from real measurement data is close to the one of identification from simulated data. Thus, as verified in the case of simulated data, the bias error is supposed to be dominant in the identification error (hypothesis 2) of paragraph 3.5), and hypothesis 1) and 3) are reasonable. So a one-step bias reduction (as described in section 3.5) is used. The measurement noise is supposed to be white gaussian, with standard deviation equal to $4.10^{-5}$, this value being computed from data measured when the mirror is stabilized (i.e. the angle value is constant).

\footnotetext{
${ }^{7}$ Moreover all the uncertain parameters or functions are identified.
}

\subsubsection{Global identification}

The algorithm described in section 4 has been implemented on the sets of data $\left\{\theta_{\mathrm{m}}^{j, k}\right\}, j=8$ : 20 , associated with $V_{j} \in[18.25 \mathrm{~V}, 31.64 \mathrm{~V}]$ : only significant data (see section 6.2) of the first $5 \mathrm{~ms}$ are considered. The initial conditions are not identified (the system is initially at rest).

After bias reduction, the following identified parameters are obtained:

$$
\begin{aligned}
I^{*}= & 1.944 \times 10^{-5}, \\
K^{*}= & 3.874 \times 10^{3}, \\
\mu_{1}^{*}= & -5.061 \times 10^{5}, \\
& \\
c^{*}= & {\left[\begin{array}{c}
0.0208 \\
-1.0252 \\
217.228 \\
9.950 \times 10^{3} \\
3.0043 \times 10^{5} \\
-4.2838 \times 10^{7}
\end{array}\right] . }
\end{aligned}
$$

As said previously, the parameters are identified up to the multiplicative constant $\mu_{0}$. To deduce the physical parameters from the identified quantities, the value of one parameter has to be known (or computed): then all the others physical quantities will be deduced from this value. For example, from the dimensions of the MEMS (see Fig. 3) and the density of silicon $\left(2.33 \times 10^{3} \mathrm{~kg} / \mathrm{m}^{3}\right)$, the physical value $\tilde{I}$ of inertia moment can be computed:

$$
\tilde{I}=2.693 \times 10^{-16} \mathrm{Nms}^{2} / \operatorname{rad} \text { (computed) }
$$




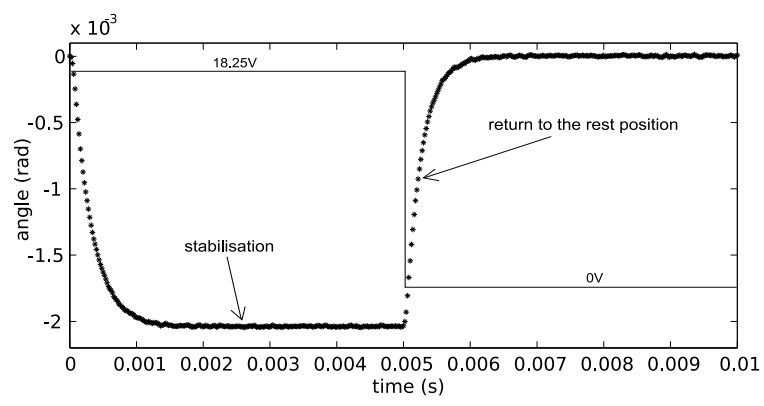

(a) $V=18.25 \mathrm{~V}<V_{\text {pullin }}^{\text {dyn }}$

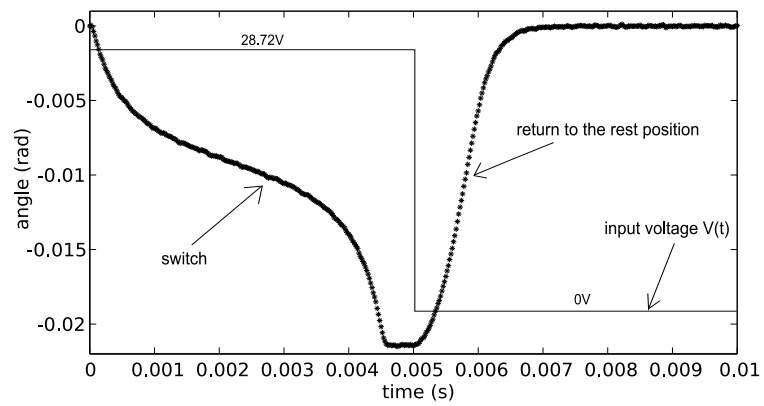

(b) $V=28.72 \mathrm{~V}>V_{\text {pullin }}^{\text {dyn }}$

Figure 9: Data $\theta_{\mathrm{m}}^{j, k}$ for different values of $V_{j}$

the deduced values of $\tilde{\mu}_{0}$ and $\tilde{K}$ are then given by:

$$
\begin{gathered}
\tilde{\mu}_{0}=\frac{\tilde{I}}{I^{*}} \mu_{0}=1.385 \times 10^{-11} \mathrm{Nm} \mathrm{s} / \mathrm{rad}, \\
\tilde{K}=\frac{\tilde{I}}{I^{*}} K^{*}=5.366 \times 10^{-8} \mathrm{~N} \mathrm{~m} / \mathrm{rad},
\end{gathered}
$$

and the same for $\mu_{1}^{*}$ and $c^{*}$.

The cumulated relative error $\mathrm{E}$ on the trajectories used for identification and the cumulated relative error $\tilde{\mathrm{E}}$ extended to all the available measurement trajectories (thus including prediction errors) are:

$$
\mathrm{E}=0.0071 \text { and } \tilde{\mathrm{E}}=0.0095 ;
$$

it highlights the good precision of the identification process.

In Fig. 10a and 10b some examples of trajectories obtained with the identified model for $V_{10}=$ $20.69 \mathrm{~V}<V_{\text {pullin }}^{\text {dyn }}<V_{18}=29.82 \mathrm{~V}$ are given. The trajectories fit well the measured data.

The identified function $k^{*}$ is given in Fig. 11 and compared $^{8}$ with its physical approximation $g$ (given

\footnotetext{
${ }^{8}$ The comparison is made up to a multiplicative coefficient such that $k^{*}(0)=g(0)$.
}

by $(70)$ ), which clearly reveals itself inadequate for deviations of $\theta$ close to $\alpha$. For illustration, an example of simulated trajectory when the function $k$ is under the form (70) is given in Fig. 12: the model does not fit with the data, contrary to the case when $k$ is fully identified (see Fig. 10b).

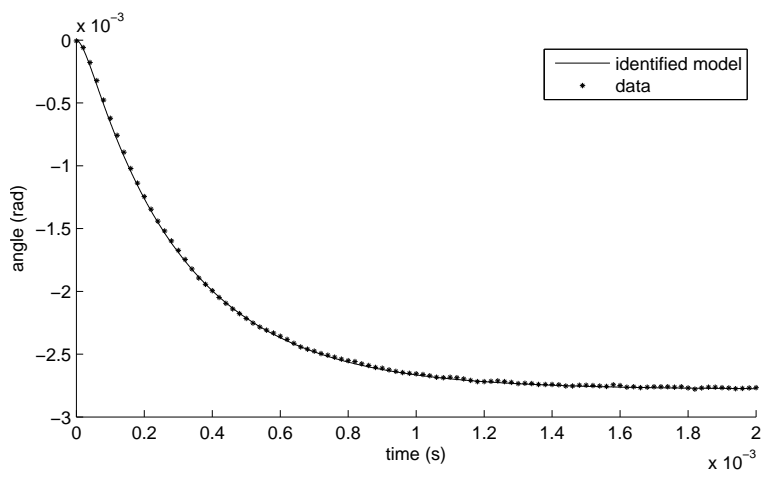

(a) $V=20.69 \mathrm{~V}$

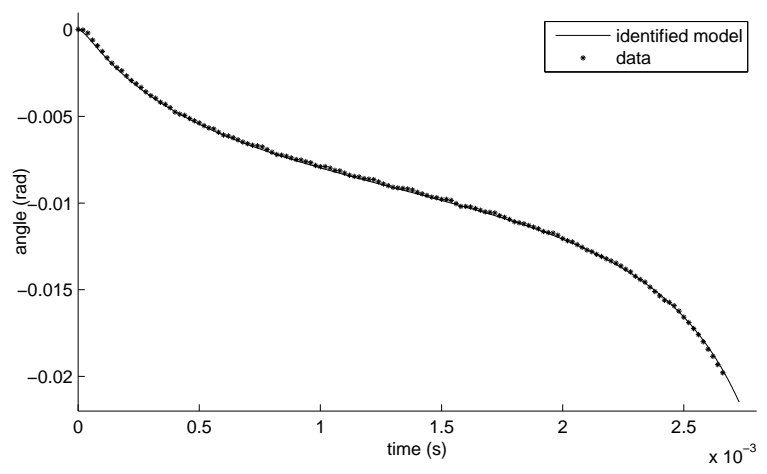

(b) $V=29.82 \mathrm{~V}$

Figure 10: Measured trajectories and the associated $\theta$ simulated from the identified model for two values of $V$

Finally, in Fig. 13 (respectively in Fig. 14), the measured and simulated stabilization/switching times (respectively angles) of the MEMS are compared, depending on the input voltage: the identified results fit well with the measured ones.

\subsubsection{Split identification}

The identification is now split into two steps. In the first one, only the part of the measured trajectories associated with $V_{j}(t)=0$ is considered, that is the return motion (to the rest position) from initial conditions reached at time where the input voltage switches to 0. During this part of the motion, no electrostatic moment acts on the system 


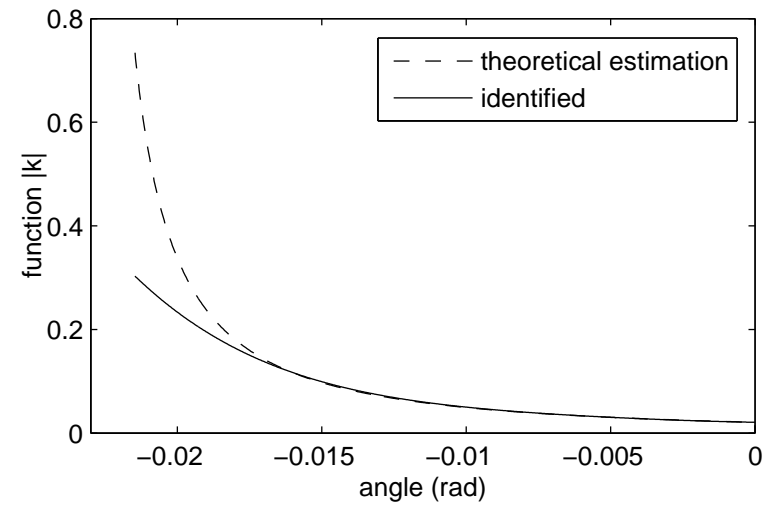

Figure 11: Absolute values of the identified $k\left(\theta^{*}\right)$ and of its theoretical estimation (70).

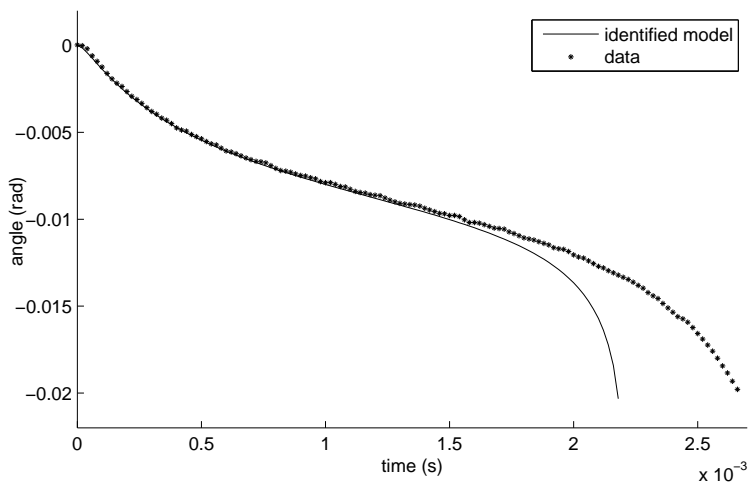

Figure 12: Measured trajectory and the associated simulated $\theta$ when $k(\theta)$ is identified under the form (70) $(V=29.82 \mathrm{~V})$.

and so, due to the weakness of the spring moment compared to the electrostatic one when $\theta$ is close to $\alpha$, the velocity $\dot{\theta}$ remains small. Consequently, the nonlinear component of $M_{f}(\theta, \dot{\theta})$ is not significantly solicited and the parameter $\mu_{1}$ is not identifiable. From these sets of data, parameters $I, K$ and, of course, the initial conditions (through parameters $a$ and $b$ ) can be identified.

In the second step, the other part of the measured trajectories (associated with $V_{j}(t)>0$ and beginning from the rest position) is used to identify the other parameters, that is $\mu_{1}$ and $c$. The previously identified parameters $I$ and $K$ are now considered as "known" parameters.

The results obtained by such a split identification are presented here-after.

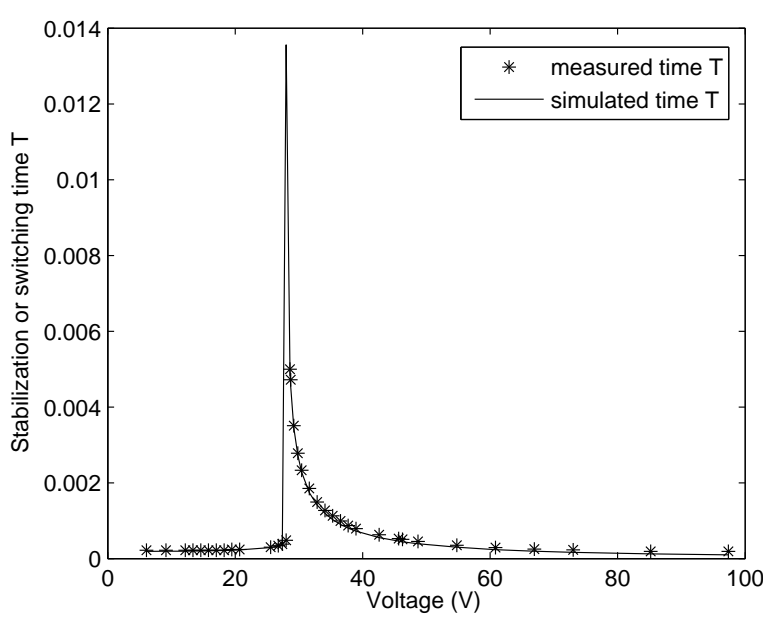

Figure 13: Stabilization (for $V<V_{\text {pullin }}^{\text {dyn }}$ ) or switching (for $V>V_{\text {pullin }}^{\text {dyn }}$ ) time $T$ for different values of V

First step: identification of $I, K, a, b$. Because $V=0$, the identification model reduces to:

$$
\left(\mathcal{H} \circ \partial_{t}^{2}\right) \theta I+\left(\mathcal{H} \circ \partial_{t}\right) \theta \mu_{0}+\mathcal{H} \theta K=h a+h b^{\prime},
$$

and the vector parameter to be identified is:

$$
\lambda:=\left(I, K, a^{T}, b^{T}\right)^{T} .
$$

For this identification, only data associated with $V_{j}=18.25 \mathrm{~V}, 19.47 \mathrm{~V}$ and $20.69 \mathrm{~V}$ are considered. The so-identified parameters are:

$$
\begin{aligned}
& I^{*}=2.077 \times 10^{-5} \\
& K^{*}=3.894 \times 10^{3} .
\end{aligned}
$$

In Fig. 15, the trajectory obtained with the identified model (83) for $V=20.69 \mathrm{~V}$ is given.

Second step: identification of $\mu_{1}, c$. The initial conditions are now equal to 0 . The parameters $I$ and $K$ are supposed to be known and equal to $I^{*}, K^{*}$ previously determined. The vector parameter to be identified is now ${ }^{9}$ :

$$
\lambda:=\left(\mu_{1}, c^{T}\right)^{T} .
$$

The identified value of $\mu_{1}$ obtained by the identification process is:

$$
\mu_{1}^{*}=-5.117 \times 10^{5} .
$$

\footnotetext{
${ }^{9}$ ad-hoc transformations of matrix $\mathrm{A}_{\mathrm{m}}$ and vector $\mathrm{b}_{\mathrm{m}}$ must be achieved, that is the components relating to $I$ and $K$ are now included in $\mathrm{b}_{\mathrm{m}}$.
} 


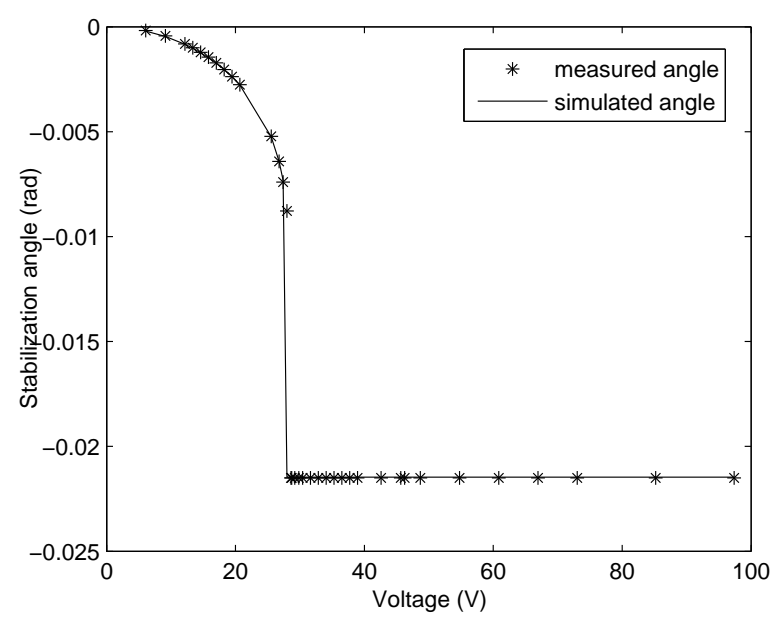

Figure 14: Stabilization angle for different values of V

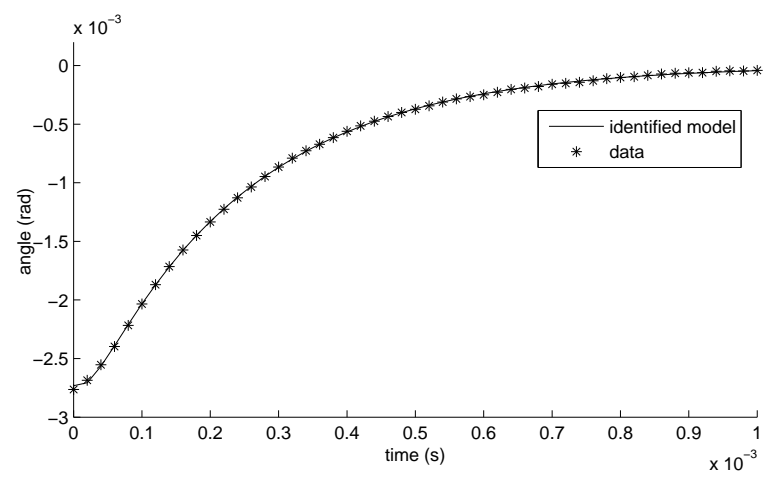

Figure 15: Measured return trajectory and the associated $\theta$ simulated from the identified model $(V=20.69 \mathrm{~V})$.

The cumulated relative error is now: $\mathrm{E}=0.0072$ (similar to section 6.3.1).

The closeness of these new identified values to the ones obtained in section 6.3.1 highlights some robustness of the method.

\subsubsection{Prediction results}

As a validation test, some results obtained in predictive situation are given here after. In Fig. 16 some data which have not been used for identification are compared to their associated trajectories predicted by the identified model. Here again, all the trajectories fit measured data with a high accuracy.

Note that the input value $V_{\max }^{2}=V_{36}^{2}=97.36^{2}$ used for the prediction test is far from the ones

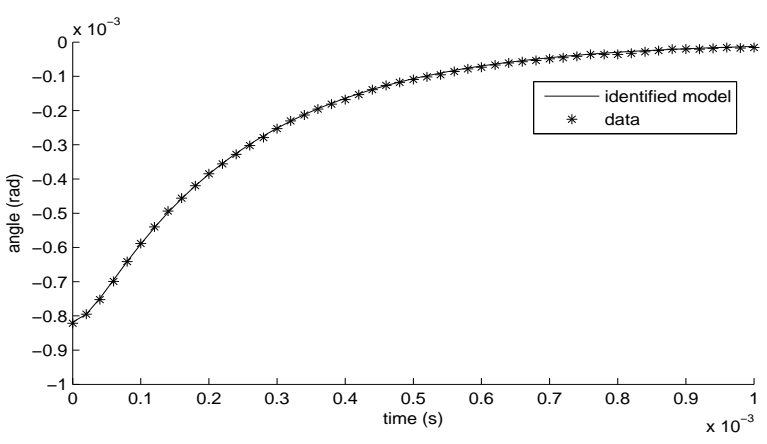

(a) return trajectory with $V=12.17 \mathrm{~V}$

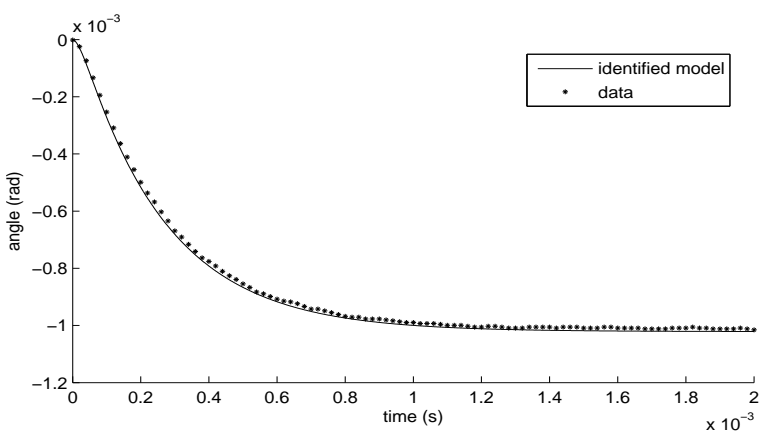

(b) $V=13.39 \mathrm{~V}$

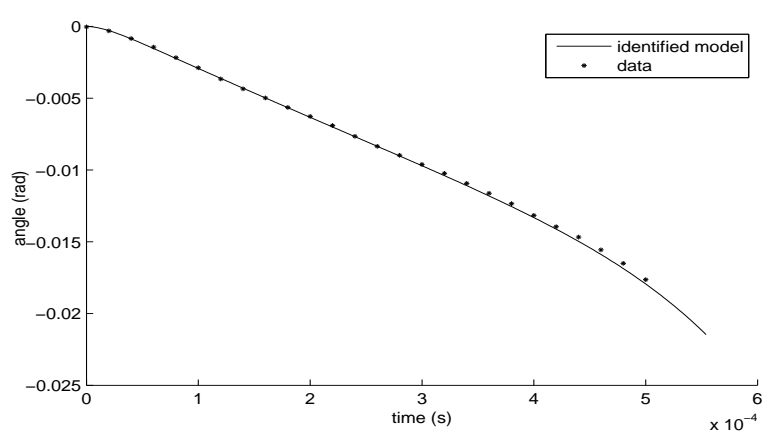

(c) $V=42.60 \mathrm{~V}$

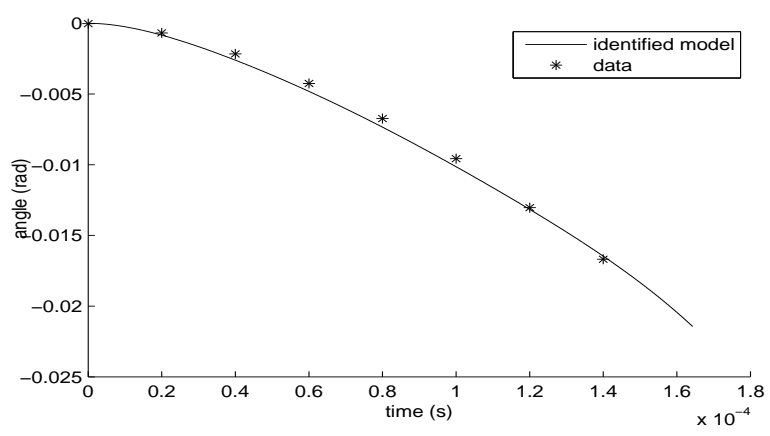

(d) $V=97.36 \mathrm{~V}$.

Figure 16: Measured trajectories and the associated $\theta$ predicted by the identified model for different values of $V$ 
used for identification (whose maximal value is $\left.V_{20}^{2}=31.64^{2}\right)$; indeed, $\frac{V_{36}^{2}}{V_{20}^{2}} \simeq 10$. In such a situation, significant identification errors on the system parameters would generate large prediction errors; in the present case, the predictive results remain good and confirm the identification accuracy.

\section{Conclusion}

In this paper, an identification method for a nonlinear dynamic model of electrostatically actuated MEMS is proposed, using operatorial prefiltering to take advantage from the linearity with respect to the parameters to be identified. This method was first validated on noised simulated data, and then applied to real measurement data. In both cases, the results are quite good, and the identified model is relevant and accurate, even in prediction situations. An iterative bias-reduction procedure was established to enhance the identification quality.

The accuracy of the so-identified model suggests several applications in the future. Namely, a high precision tension reference can be built by controlling the micro-mirror around its semi-stable bifurcation point.

\section{A. Analysis of model (3)}

When $V$ is constant, by considering the electrostatic and spring moments given in Fig. 17, equilibrium states are obtained when the electrostatic and the spring moments are balanced. As highlighted in Fig. 17, there exists a "pull-in" voltage $V_{\text {pullin }}$ such that:

- if $V<V_{\text {pullin }}$, there are two equilibrium points. It can be shown that the first one is stable and the other is unstable $((\theta, \dot{\theta})$ stabilizes on the stable one, see Fig. 9a);

- if $V=V_{\text {pullin }}$, there is one unstable equilibrium point;

- if $V>V_{\text {pullin }}$, there is not any equilibrium point: the system "switches" ( $\theta$ goes to the saturation value $\alpha$, see Fig. 9b).

In the case where friction moments are neglected, the three associated phase portraits (that is the trajectories of $(\theta, \dot{\theta}))$ are given in Fig. 18.

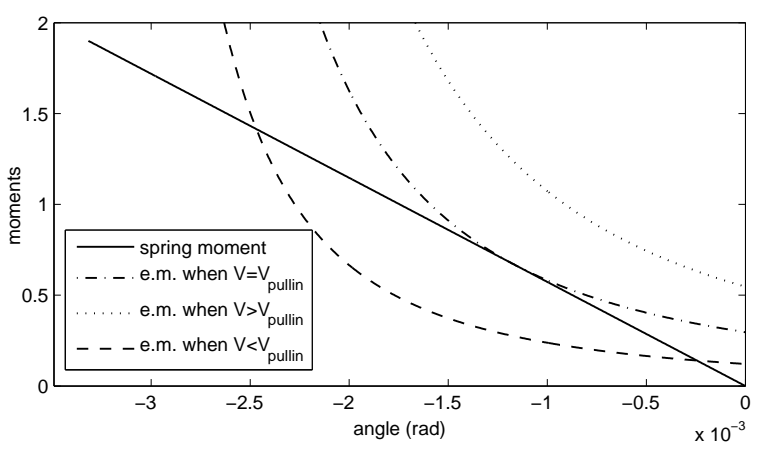

Figure 17: Absolute values of spring moment and electrostatic moment (e.m.) for different values of $V$.

In the case where $V<V_{\text {pullin }}$, three different behaviors can occur depending on the initial point $\left(\theta_{0}, \dot{\theta}_{0}\right)$ (here taken equal to $\left.(0,0)\right)$ and the equilibrium ones (see Fig. 19). If the stable equilibrium point is far enough from the initial condition, then $\theta$ goes to $\alpha$ (the system switches), whereas in the opposite case, $\theta$ does not reach the switching value. In that sense, the limit case is a bifurcation whose associated "dynamic pull-in" value of $V$ is denoted by $V_{\text {pullin }}^{\text {dyn }}<V_{\text {pullin }}$.

Remark 13. The analytical expressions of $V_{\text {pullin }}^{\text {dyn }}$ and $V_{\text {pullin }}$ are in general impossible to compute exactly because they depend on the expression of the electrostatic moment (non necessarily known), and so on the configuration of the physical system. In some cases however, their expressions can be computed. For example, in the case of inclined electrodes (Camon and Larnaudie (2000)), the expression of the electrostatic moment is fairly simple:

$$
M_{e}(\theta, V)=-\frac{k V^{2}}{(\alpha-\theta)^{2}},
$$

with $k>0$ depending on the physical parameters. When the viscous friction forces are neglected, the analytical expressions of $V_{\text {pullin }}^{\text {dyn }}$ and $V_{\text {pullin }}$ are then given by:

$$
V_{\text {pullin }}=\sqrt{-\frac{8 \alpha^{3} K}{27 k}}, V_{\text {pullin }}^{\text {dyn }}=\sqrt{-\frac{\alpha^{3} K}{4 k}},
$$

and the maximal stabilization angle from initial condition $(0,0)$ (reached for $\left.V=V_{\text {pullin }}^{\text {dyn }}\right)$ is equal to $\frac{\alpha}{2}$.

Remark 14. When the viscous friction forces are neglected, it can be shown that whatever the 


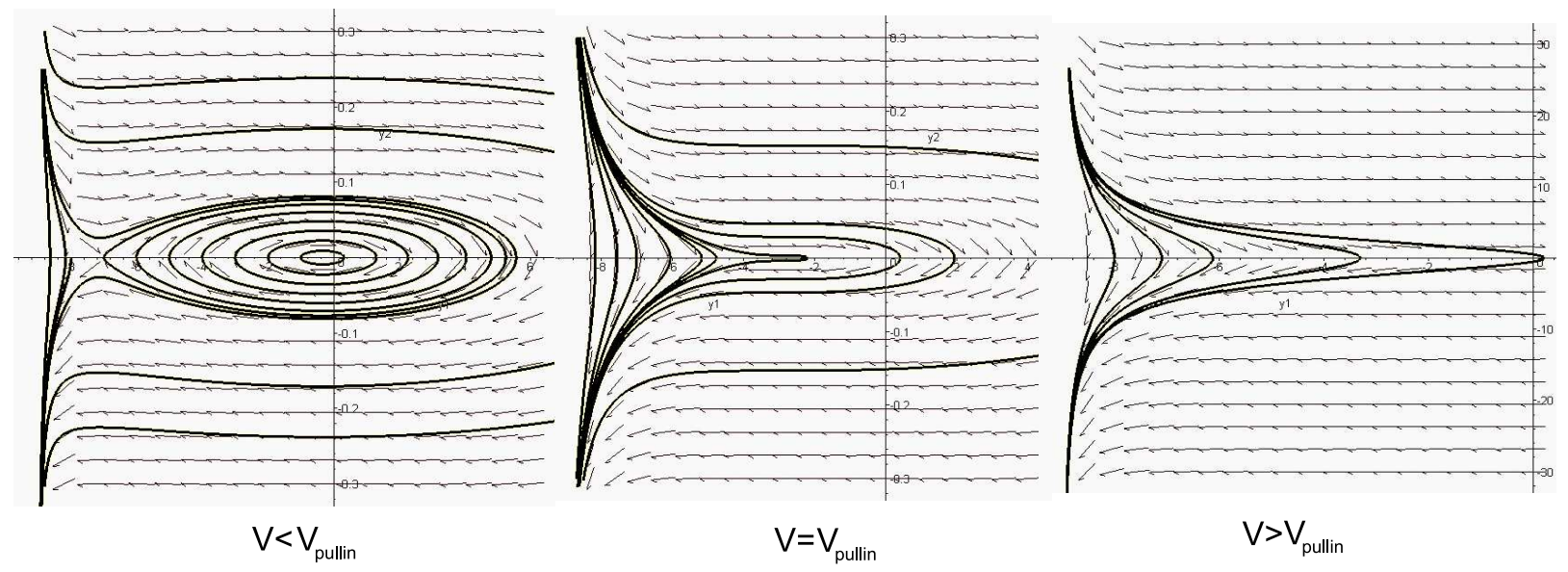

Figure 18: Phase portraits according to the value of $V$ (the viscous friction forces are here neglected).

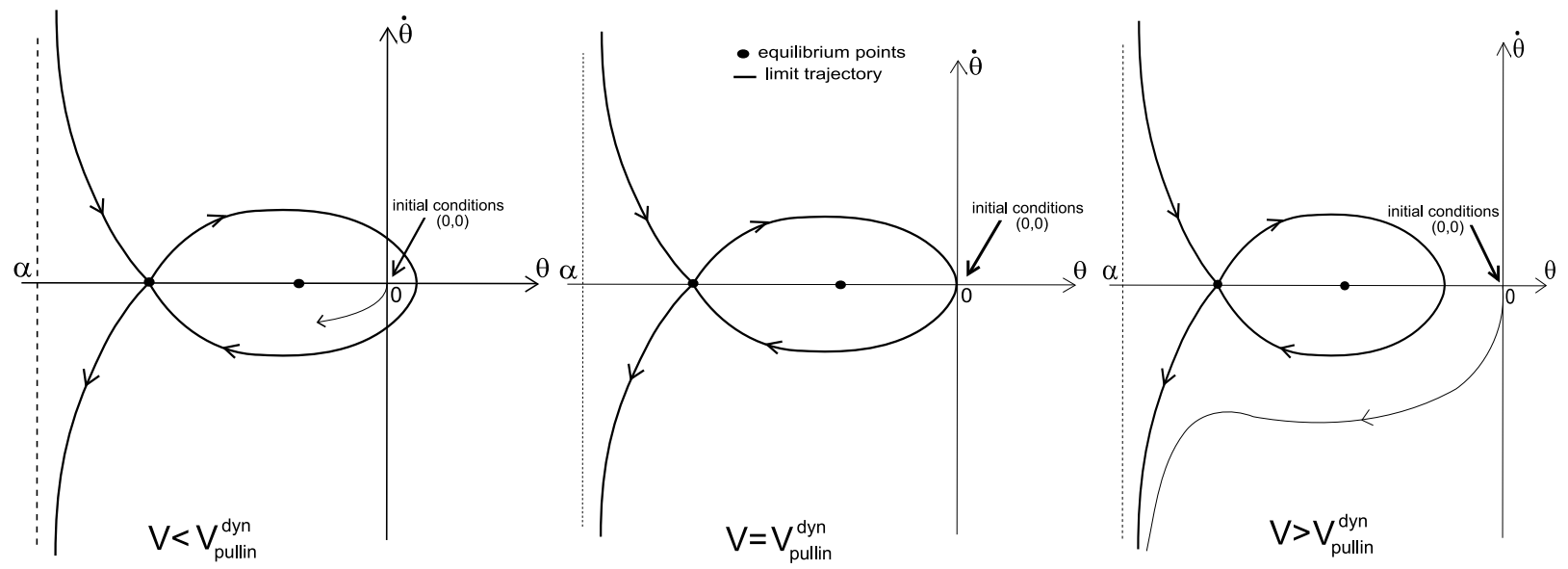

Figure 19: Trajectories according to the value of $V<V_{\text {pullin }}$.

electrostatic moment expression is, the "switching time" $T$ such that $\theta(T)=\alpha$ can be expressed for any $V>V_{\text {pullin }}^{\text {dyn }}$ by:

$$
T_{\text {switch }}(V)=\int_{0}^{\alpha} \frac{1}{-\sqrt{V^{2} \kappa(\theta)-\frac{1}{2} K \theta^{2}}} d \theta,
$$

where $\kappa(\theta)$ is the antiderivative of $k(\theta)$ such that $\kappa\left(\theta_{0}\right)=\frac{1}{2 V^{2}}\left(I \theta_{1}^{2}+K \theta_{0}^{2}\right)$.

\section{B. Equivalence between models $(3,5)$ and (15)}

Consider the two models $(3,5)$ and (15).
Let first suppose $\theta$ is solution of $(3,5)$. After two integrations between 0 and $t$ of equation (3), it follows:

$$
\begin{gathered}
I \theta(t)+\mu_{0} \partial_{t}^{-1} \theta(t)+\partial_{t}^{-1} w(\theta)(t)+K \partial_{t}^{-2} \theta(t)= \\
V^{2} \partial_{t}^{-2} k(\theta)(t)+I \theta_{0}+\left(I \theta_{1}+\mu_{0} \theta_{0}+w\left(\theta_{0}\right)\right) t
\end{gathered}
$$

where $w$ is the antiderivative of $v$ such that $w(0)=$ 0 and $\partial_{t}^{-2}:=\partial_{t}^{-1} \circ \partial_{t}^{-1}$. In the sense of distributions:

$$
\partial_{t} \circ \partial_{t}^{-1}=1, \partial_{t} \mathbf{1}_{\mathbb{R}^{+}}=\delta \text { and } \partial_{t} \delta=\delta^{\prime}
$$

thus, composition of (91) with $\partial_{t}^{2}$ leads to (15) with: 
Conversely, let show that if $\theta$ is solution of (15) then $\theta$ is solution of $(3,5)$.

If $\theta$ is solution of (15), then $\theta$ obviously verifies equation (3) for all $t>0$. By denoting

$$
\theta_{p}=\partial_{t} \theta-\frac{b}{I} \delta
$$

equation (15) can be written under the form:

$$
\left\{\begin{array}{lr}
\partial_{t} \theta_{p}=\frac{1}{I}\left(V^{2} k(\theta)-\mu_{0} \theta_{p}-K \theta\right) & +\frac{1}{I}\left(a-\mu_{0} \frac{b}{I}\right) \delta \\
\partial_{t} \theta=\theta_{p}+\frac{b}{I} \delta, & -\frac{1}{I} \partial_{t} w(\theta),
\end{array}\right.
$$

which gives after composition with $\partial_{t}^{-1}$ and for $t=0$ :

$$
\left\{\begin{array}{l}
\theta_{p}(0)=\frac{1}{I}\left(a-\mu_{0} \frac{b}{I}-w(\theta(0))\right), \\
\theta(0)=\frac{b}{I}
\end{array}\right.
$$

that is, with (93): $\theta(0)=\theta_{0}$ and $\theta_{p}(0)=\theta_{1}$. Then it follows: $\theta_{p}=\partial_{t} \theta-\theta(0) \delta$, that is $\theta_{p}=\dot{\theta}$ and so $\dot{\theta}(0)=\theta_{1}$

\section{Computation of the components of $A_{m}$ and $b_{m}$}

If $\theta_{\mathrm{m}}, \mathbf{w}\left(\theta_{\mathrm{m}}\right)$ and $\mathbf{k}\left(\theta_{\mathrm{m}}\right)$ are assumed to be some piecewise polynomial functions of degree 3 , the components of $\mathrm{A}_{\mathrm{m}}$ and $\mathrm{b}_{\mathrm{m}}$ can be analytically computed. These computations only necessitate to know how to compute $\mathcal{H} u, \mathcal{H} \circ \partial_{t} u$ and $\mathcal{H} \circ \partial_{t}^{2} u$ when $u$ is a cubic spline.

Let denote by $\mathcal{G}$ the operator $\sigma\left(\partial_{t}+\sigma\right)^{-1}$ and $\mathcal{H}=\sigma^{2}\left(\partial_{t}+\sigma\right)^{-2}=\mathcal{G} \circ \mathcal{G}$. Let $u$ be a piecewise polynomial function of degree 3 , that is a function of the form:

$$
u(t)=\sum_{n=0}^{N-1} \sum_{k=0}^{3} u_{k, n}\left(t-t_{n}\right)^{k} \mathbf{1}_{\left[t_{n}, t_{n+1}[\right.}(t) .
$$

\section{C.1. Computation of $\mathcal{H} u$ and $\mathcal{G} u$}

For any function $u$ :

$$
\begin{aligned}
& (\mathcal{G} u)(t)=\sigma \int_{0}^{t} e^{-\sigma(t-s)} u(s) d s, \\
& (\mathcal{H} u)(t)=\sigma^{2} \int_{0}^{t} \int_{0}^{s} e^{-\sigma(t-r)} u(r) d r d s,
\end{aligned}
$$

and so:

$$
\begin{array}{cc}
(\mathcal{G} u)\left(t_{l}\right)= & e^{-\sigma \Delta t}(\mathcal{G} u)\left(t_{l-1}\right) \\
& +\sigma e^{-\sigma t_{l}} \int_{t_{l-1}}^{t_{l}} e^{\sigma s} u(s) d s, \\
(\mathcal{H} u)\left(t_{l}\right)= & e^{-\sigma \Delta t}(\mathcal{H} u)\left(t_{l-1}\right) \\
& +\sigma^{2} e^{-\sigma t_{l}} \int_{t_{l-1}}^{t_{l}} \int_{0}^{s} e^{\sigma r} u(r) d r d s .
\end{array}
$$

If $u$ is defined by (97), then:

$$
\begin{array}{r}
\int_{t_{l-1}}^{t_{l}} e^{\sigma s} u(s) d s=\sum_{k=0}^{3} u_{k, l-1} e^{\sigma t_{l-1}} I_{k}(\Delta t), \\
\int_{t_{l-1}}^{t_{l}} \int_{0}^{s} e^{\sigma r} u(r) d r d s=\Delta t \sum_{n=0}^{l-2} e^{\sigma t_{n}} \sum_{k=0}^{3} u_{k, n} I_{k}(\Delta t) \\
+e^{\sigma t_{l-1}} \sum_{k=0}^{3} u_{k, l-1} \int_{0}^{\Delta t} I_{k}(z) d z .
\end{array}
$$

where $I_{k}(x)=\int_{0}^{x} e^{\sigma r} r^{k} d r$. The expression of $I_{k}(x)$ is given by the following induction:

$$
\left\{\begin{array}{l}
I_{k}(x)=x^{k} \frac{e^{\sigma x}}{\sigma}-\frac{k}{\sigma} I_{k-1}(x), \\
I_{0}(x)=\frac{1}{\sigma}\left(e^{\sigma x}-1\right),
\end{array}\right.
$$

and so it follows:

$$
\left\{\begin{array}{l}
\int_{0}^{\Delta t} I_{k}(z) d z=\frac{1}{\sigma} I_{k}(\Delta t)-\frac{k}{\sigma} \int_{0}^{\Delta t} I_{k-1}(z) d z \\
\int_{0}^{\Delta t} I_{0}(z) d z=\frac{1}{\sigma}\left(I_{0}(\Delta t)-\Delta t\right) .
\end{array}\right.
$$

\section{C.2. Computation of $\left(\partial_{t} \circ \mathcal{H}\right) u$ and $\left(\partial_{t}^{2} \circ \mathcal{H}\right) u$}

Because $\mathcal{H} u=\sigma\left(\partial_{t}+\sigma\right)^{-1} \circ \mathcal{G} u=\sigma^{2}\left(\partial_{t}+\sigma\right)^{-2} u$, it follows:

$$
\begin{aligned}
& \left(\partial_{t} \circ \mathcal{H}\right) u=\sigma \mathcal{G} u-\sigma \mathcal{H} u, \\
& \left(\partial_{t}^{2} \circ \mathcal{H}\right) u=\sigma^{2} u-\sigma^{2} \mathcal{H} u-2 \sigma\left(\partial_{t} \circ \mathcal{H}\right) u .
\end{aligned}
$$

\section{References}

Adams, R., Fournier, J., 2003. Sobolev Spaces. Academic Press.

Ben-Israel, A., Greville, T., 2003. Generalized inverses: Theory and applications. Springer Verlag, New York, USA.

Bingulac, S., Sinha, N., 1989. On the identification of continuous-time systems from the samples of input-output data. In: Proceedings of the 7th International Conference on Mathematical and Computer Modeling. Chicago (USA), pp. 231-239. 
Bryzek, J., Abbott, H., Flannery, A., Cagle, D., Maitan, J., Ventures, B., Fremont, C., 2003. Control issues for mems. In: Proceedings of the 42nd IEEE Conference on Decision and Control. Vol. 3. pp. 3039-3047.

Camon, H., Ganibal, C., Rapahoz, N., Trzmiel, M., Pisella, C., Martinez, C., Valette, S., 2008. Solving functional reliability issue for an optical electrostatic switch. Microsystem Technologies 14 (7), 919-923.

Camon, H., Larnaudie, F., 2000. Fabrication, simulation and experiment of a rotating electrostaticsilicon mirror with large angular deflection. In: The 13th Annual International Conference on Micro Electro Mechanical Systems. Miyazaki (Japan), pp. 645-650.

Cichalewski, W., Napieralski, A., Camon, H., Estibals, B., 2003. Study of mems micro mirror pull-in voltage and angle, for inclined and combined (inclined/flat) electrode structure. In: 10th International Conference on Mixed Design of Integrated Circuits and Systems (MIXDES'2003). Lodz (Pologne), pp. 466-469.

Daqaq, M., Reddy, C., Nayfeh, A., 2006. Input-shaping control of nonlinear mems. In: Proceedings of the IMACXXII Conference and Exposition on Structural Dynamics. Vol. 54. pp. 167-179.

Garnier, H., Wang, L., 2008. Identification of continuoustime models from sampled data. Springer.

Garnier, H., Young, P., 2004. Time-domain approaches to continuous-time model identification of dynamical systems from sampled data. In: Proceedings of the 2004 American Control Conference. Vol. 1. Boston.

Jemni, A., Trigeassou, J. C., 1996. A comparative study of the deterministic accuracy of two identification methods for continuous systems. In: In Proceedings, IEEE SMC CESA96 IMACS Symposium on Modelling, Analysis and Simulation. Vol. 1. Lille (France), p. 488493.

Liao, K.-M., Wang, Y.-C., Yeh, C.-H., Chen, R., 2004. Closed-loop adaptive control for torsional micromirrors. In: Proceedings of SPIE - In EL-FATATRY, AYMAN (editor): MOEMS and Miniaturized Systems IV. Vol. 5346. Bellingham, WA,, pp. 184-192.

Ljung, L., 1987. System identification: theory for the user. Prentice-Hall Englewood Cliffs, NJ.

Mahata, K., Garnier, H., 2005. Direct identification of continuous-time errors-in-variables models. In: Preprints of the 16th Triennal IFAC World Congress on Automatic Control. Prague (Czech Republic), pp. 3-8.

Mahata, K., Garnier, H., 2006. Identification of continuoustime errors-in-variables models. Automatica 42 (9), 14771490.

Sagara, S., Zhao, Z., 1990. Numerical integration approach to on-line identification of continuous-time systems. Automatica 26 (1), 63-74.

Saha, D., Rao, B., Rao, G., 1982. Structure and parameter identification in linear continuous lumped systems the Poisson moment functional approach. International Journal of Control 36 (3), 477-491.

Sane, H., Yazdi, N., Mastrangelo, C., 2005. Robust control of electrostatic torsional micromirrors using adaptive sliding-mode control. In: Proceedings of SPIE - Photonics West 2005. Vol. 5719. San Jose, CA, pp. 115-126.

Schumaker, L., 2007. Spline functions: Basic Theory. Cambridge Mathematical Library.

Sinha, N., Rao, G., 1991. Identification of continuous-time systems: Methodology and computer implementation. Microprocessor-based \& Intelligent Systems Engineering, Kluwer Academic Publishers, Dordrecht.
Söderström, T., Mahata, K., 2002. On instrumental variable and total least squares approaches for identification of noisy systems. International Journal of Control 75 (6), 381-389.

Vagia, M., Nikolakopoulos, G., Tzes, A., 2008. Design of a robust PID-control switching scheme for an electrostatic micro-actuator. Control Engineering Practice 16 (11), 1321 - 1328.

Welsh, J., Goodwin, G., Garnier, H., 2007. A simple method for bias reduction in time domain least squares parameter estimation. In: 3rd IFAC Symposium on System, Structure and Control, SSSC07. Foz do Iguau(Brasil).

Yosida, K., 1980. Functional analysis. Springer-Verlag, Berlin.

Young, P., 1965a. Process parameter estimation and self adaptive control. In: Proceedings of the Second IFAC Symposium on the Theory of Self-adaptive Control Systems. Teddington (England), pp. 118-140.

Young, P., 1965b. The determination of the parameters of a dynamic process. Radio and Electronic Engineering (Journal of IERE) 29, 345-361.

Young, P., 1970. An instrumental variable method for realtime identification of a noisy process. Automatica 6 (2), 271-287.

Young, P., Garnier, H., 2006. Identification and estimation of continuous-time, data-based mechanistic (DBM) models for environmental systems. Environmental modelling \& software 21 (8), 1055-1072.

Zhu, G., Penet, J., Saydy, L., 2007. Modeling and control of electrostatically actuated MEMS in the presence of parasitics and parametric uncertainties. Journal of Dynamic Systems, Measurement, and Control 129, 786-794. 